\title{
One-loop CHY-integrand of bi-adjoint scalar theory
}

\author{
Bo Feng ${ }^{a, b}$ and Chang $\mathbf{H u}^{a, 1}$ \\ ${ }^{a}$ Zhejiang Institute of Modern Physics, Zhejiang University, \\ Zheda Road No. 38, Hangzhou, 31002\%, P.R. China \\ ${ }^{b}$ Center of Mathematical Science, Zhejiang University, \\ Zheda Road No. 38, Hangzhou, 310027, P.R. China \\ E-mail: fengbo@zju.edu.cn, isiahalbert@126.com
}

ABSTRACT: In this paper, the one-loop CHY-integrands of bi-adjoint scalar theory has been reinvestigated. Differing from previous constructions, we have explicitly removed contributions from tadpole and massless bubbles when taking the forward limit of corresponding tree-level amplitudes. The way to remove those singular contributions is to exploit the idea of "picking poles", which is to multiply a special cross ratio factor with the role of isolating terms having a particular pole structure.

Keywords: Scattering Amplitudes, Field Theories in Higher Dimensions, Field Theories in Lower Dimensions

ARXIV EPRINT: 1912.12960

\footnotetext{
${ }^{1}$ Corresponding author.
} 


\section{Contents}

1 Introduction 1

2 Backgrounds 3

2.1 Integrate rules 3

$\begin{array}{ll}2.2 & \text { Effective Feynman diagram }\end{array}$

2.3 Picking out poles 8

3 One loop 10

$3.1 \pi=\rho \quad 12$

$3.2 \pi=\rho^{T} \quad 16$

$\begin{array}{lll}3.3 & \text { For general orderings } \pi \text { and } \rho & 20\end{array}$

$\begin{array}{lll}3.4 & \text { Example } & 25\end{array}$

3.4.1 The first example 26

$\begin{array}{ll}3.4 .2 & \text { The second example }\end{array}$

4 Conclusion $\quad 29$

\section{Introduction}

In recent years, a novel formulism for tree-level amplitudes of various theories has been proposed by Cachazo, He and Yuan [CHY] in a series of papers [1-5]. The formula is given as an integral over the moduli space of Riemann spheres

$$
\mathcal{A}_{n}=\int \frac{\left(\prod_{i=1}^{n} d z_{i}\right)}{\operatorname{vol}(\operatorname{SL}(2, \mathbb{C}))} z_{i j} z_{j k} z_{k i} \prod_{a \neq i, j, k} \delta\left(\mathcal{E}_{a}\right) \cdot I\left(z_{1}, \cdots, z_{n}\right)
$$

where $z_{i}$ are puncture locations of (the) $i$-th external particles, and the denominator $\operatorname{vol}(\operatorname{SL}(2, \mathbb{C}))$ comes from the Möbius invariance. i.e., the transformation $z_{i} \rightarrow \frac{a z_{i}+b}{c z_{i}+d}$ with $a d-b c \neq 0$. The $\mathcal{E}$ 's are the scattering equation defined as

$$
\mathcal{E}_{a} \equiv \sum_{b \neq a} \frac{s_{a b}}{z_{a}-z_{b}}=0, a=1,2, \ldots, n
$$

with $s_{a b} \equiv\left(k_{a}+k_{b}\right)^{2}$ being the Mandelstam invariants. As shown in (1.1), the CHY formalism includes two parts: the integration measure with $\delta$ functions of scattering equations, which is universal for all theories, and formulating different CHY-integrands $I$ for different theories. For a theory with $n$ particles, there are $(n-3)$ ! solutions to these scattering equations. A proof of this construction for bi-adjoint scalar theory and Yang-Mills theory has been provided by Dolan and Goddard in [6]. Working out all $(n-3)$ ! solutions of 
scattering equation is very burdensome even at small value of $n$. In general there is no effective method to solve these equations analytically if $n \geq 6$. By exploiting computational algebraic geometry method, several works have appeared [7-13], such as the companion matrix, the Bezoutian matrix, the elimination theorem, etc. An alternative way is given in $[14,15]$ by mapping the problem to the result of bi-adjoint $\phi^{3}$ theory which is already known. By applying the generalized KLT relation and Hamiltonian decomposition of a certain 4-regular graph, one can read out the result directly without solving the scattering equations. In [16-18], an integration rule has been presented to read out Feynman diagrams for CHY-integrands containing only single poles. For more general CHY-integrand ${ }^{1}$ with higher poles, we can use the cross ratio identities [19] to reduce the degree of poles one by one until all poles are single poles, then the integration rule can be applied.

Having established the CHY formalism for tree-level amplitudes, it is natural to ask the generalization to loop levels. Many works have been done in this direction [18, 21-36]. In $[24,25]$, using the ambitwistor string theory, through the global residue theorem, Geyer, Mason, Monteiro and Tourkine have written down a beautiful one-loop CHY formulism (i.e., the loop scattering equations and loop CHY-integrands) for various theories like superYang-Mills theory, n-gon, supergravity, pure Yang-Mills and gravity theory. ${ }^{2}$ Integration rule has been generalized to loop level in [18] and the CHY-integrand of bi-adjoint scalars has been discussed. In [26], the idea that one loop integrands coming from the forward limit of tree-level amplitudes has been presented, where the bi-adjoint scalar theory has been investigated as an example. Singular solutions related to the forward limits have also been clarified. The role of regular and singular solutions of scattering equations has been further illustrated in [27].

Above works pointed out two important issues for the one-loop construction in the CHY frame. Firstly, when taking the forward limit, there are singularities we should deal with carefully. Secondly, as emphasized in [27], when we talk about the one-loop integrands of a given theory, we should think it in the sense of equivalent class, i.e., expressions in the same class can be different by scaleless parts. In this paper, we will suggest a new approach to treat the singularities related to the forward limit. The way to remove those singular contributions is to exploit the idea of "picking poles", which is to multiply a special cross ratio factor with the role of isolating terms having a particular pole structure. As an application of our new strategy, one-loop CHY-integrands of bi-adjoint scalar theory will be re-investigated.

The plan of the paper is follows. In the next section we provide a lightning review of some necessary backgrounds for this paper, including the integration rules that enable us to evaluate amplitude from a given CHY-integrand, the conception and some properties of effective Feynman diagrams, and the cross ratio factor that allow us to pick up terms containing a given pole structure. In section three we reinvestigate the bi-adjoint scalar theory. Firstly we review the construction idea given in $[18,26]$ and the relation between loop diagrams and tree diagrams through the cutting and gluing process. Based on the

\footnotetext{
${ }^{1}$ A special case, i.e., Yang-Mills amplitudes, has been discussed in [20].

${ }^{2}$ Although we will focus on one-loop case only in this paper, it is worth to mention that using the same idea, some two loop constructions are shown in [37-40].
} 
connection, we construct the one-loop CHY-integrand for two special cases, i.e., two PTfactors have same orderings or opposite orderings. Combining results of above two special cases and the effective Feynman diagram of corresponding tree-level amplitudes, we give a general construction of one-loop CHY-integrand for two arbitrary PT-factors. Finally, in the section four, we give brief summary and discussion.

\section{Backgrounds}

In this section, we will review some known results, which are useful for our construction of one-loop CHY-integrands of bi-adjoint scalar theory.

\subsection{Integrate rules}

In this subsection, we will review how to write down the analytic expression for the amplitudes given by

$$
A=\int d \Omega_{C H Y} I\left(z_{1}, \cdots, z_{n}\right)
$$

in the CHY formalism. A generic CHY-integrand can be given by the sum of following terms

$$
\mathcal{I}=\frac{1}{\prod_{1 \leq i<j \leq n} z_{i j}^{\beta_{i j}}},
$$

where $z_{i j} \equiv z_{i}-z_{j}$ and $\beta_{i j}$ 's can be any integer numbers. ${ }^{3}$ The Möbius invariance requires that $\sum_{j<i} \beta_{j i}+\sum_{j>i} \beta_{i j}=4$. For each term like (2.2), we can define the pole index of a subset $A_{i} \subset\{1,2, \ldots, n\}$ as

$$
\chi\left(A_{i}\right) \equiv L\left[A_{i}\right]-2\left(\left|A_{i}\right|-1\right),
$$

where $\left|A_{i}\right|$ is the number of external particles inside the subset $A_{i}$ and $L\left[A_{i}\right]$ is the linking number which is given by

$$
L\left[A_{i}\right]=\sum_{a<b ; a, b \in A_{i}} \beta_{a b} .
$$

It is worth to notice that the subset $A_{i}$ and its complement subset $\bar{A}_{i}$ have the same pole index by the Möbius invariance condition. When the pole index $\chi \geq 0$, the amplitude can have terms with poles like $\frac{1}{s_{A_{i}}^{\chi\left[A_{i}\right]+1}}$, where

$$
s_{A_{i}}=\left(\sum_{a \in A_{i}} k_{a}\right)^{2}=\left(\sum_{b \in \bar{A}_{i}} k_{b}\right)^{2} .
$$

Now we show the algorithm ${ }^{4}$ to read out the corresponding Feynman diagrams from the given CHY-integrands (2.2).

\footnotetext{
${ }^{3}$ When $\beta<0$, it is numerator.

${ }^{4}$ There are several algorithms in the literatures, see related references $[3,14,16-20,41-46]$. In this paper, we review these methods which are suitable for our purpose.
} 


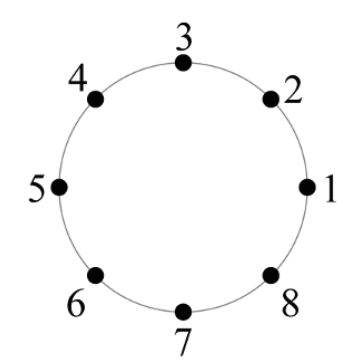

(a)

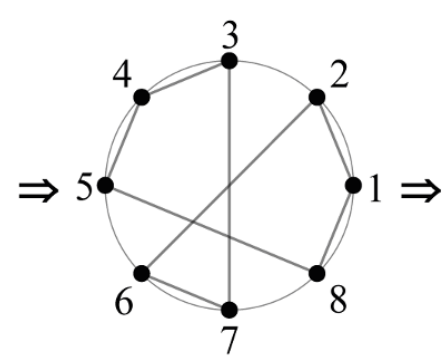

(b)

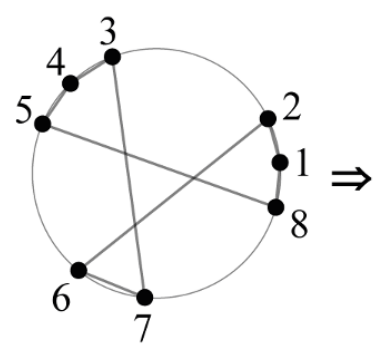

(c)

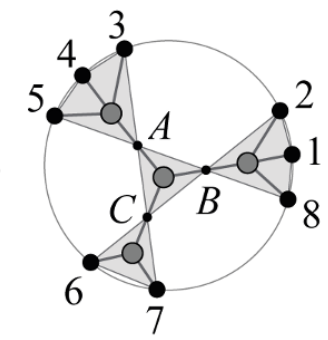

(d)

Figure 1. The procedure to obtain the effective Feynman diagram for the ordering $\pi=(12345678)$ and $\rho=(12673458)$.

Let us start from the simplest case, i.e., the CHY-integrand $I$ is given by the product of two Park-Taylor factors

$$
I\left(z_{1}, \cdots, z_{n}\right)=P T(\pi) \times P T(\rho)
$$

where $\pi, \rho$ are two orderings of external particles and for the given ordering $\alpha$, the ParkTaylor factor is defined by

$$
P T(\pi) \equiv \frac{1}{\left(z_{\pi(1)}-z_{\pi(2)}\right)\left(z_{\pi(2)}-z_{\pi(3)}\right) \cdots\left(z_{\pi(n-1)}-z_{\pi(n)}\right)\left(z_{\pi(n)}-z_{\pi(1)}\right)},
$$

which is obviously cyclic invariance. For this particular case (2.6), [3] shows that the result is given by the sum of the collection of all trivalent scalar diagrams that can be regarded both as $\pi$-color-ordered and $\rho$-color-ordered, where each diagram's contribution is given by the product of its propagators up to a sign. To make thing clear, let us present one example with $\pi=(12345678)$ and $\rho=(12673458)$ as following:

- (a) First, we draw a disk with $n$ nodes sitting on the boundary in the ordering $\pi$ (see graph (a) of figure 1). Then we link $n$ nodes together with a loop of line segments according to the ordering $\rho$ (see graph (b) of figure 1). It is worth to notice that the closed $\rho$ loop will form many small regions by its intersections as shown in the graph (b), where there are two pentagons, one quadrilateral and one triangle. If there are small regions sharing same edge, we need to pull nodes belonging to the same region together until any two adjacent polygons share at most one common vertex as shown in the graph (c) of figure 1.

- (b) Having obtained the valid intersection pattern as shown in graph (c), we construct the effective Feynman diagram as following. First we put a vertex at the middle of each small polygon (such a vertex is called the "effective vertex"). Then we connect each vertex with external nodes of the same polygon. Finally we connect two effective vertexes together if their polygons share a common point (such a line is called "effective propagator"). The achieved figure is the effective Feynman diagram as shown in graph (d) of figure 1. 
- (c) Having the effective Feynman diagram, we can easily read out the analytic expression by noticing that each vertex with $m$ legs represents all possible Feynman diagrams with only cubic vertexes and $m$ external legs having this ordering. For this example, there are four vertexes. For the vertex connecting to nodes $1,2,8$, it gives $\frac{1}{s_{12}}+\frac{1}{s_{18}}$. Similar understanding for other three effective vertexes. Multiplying contributions from these four vertexes as well as effective propagators connecting these vertexes, we get the final result up to a sign

$$
\frac{1}{s_{128}} \frac{1}{s_{345}} \frac{1}{s_{67}} \times\left(\frac{1}{s_{12}}+\frac{1}{s_{18}}\right) \times\left(\frac{1}{s_{34}}+\frac{1}{s_{45}}\right) .
$$

Having reviewed the construction of effective Feynman diagrams up to a sign, we discuss the overall sign given by $(-1)^{n-3+n_{\text {flip }}(\pi \mid \rho)}$, where $n_{\text {flip }}$ is determined as following [3]:

- (a) At the first step, we merge consecutive nodes both in the orderings $\pi$ and $\rho$, until there is no more merging can be done. For example, with $\pi=(123456789)$ and $\rho=(128946573)$, we obtain $\pi[1]=\left(P_{12} 34567 P_{89}\right)$ and $\rho[1]=\left(P_{12} P_{89} 46573\right)$.

- (b) Next, we flip two nearby nodes in the ordering $\rho[1]$ if there two nodes are also nearby in the ordering $\pi[1]$ but with the reversing ordering. For example, pairs $\left(P_{12} P_{89}\right)$ and $(65)$ in $\rho[1]$ should be flipped. With such an operation, we get contribution $(-)^{m}$ where $m$ is number of pairs doing the flip. With above example, we have $\pi[2]=\left(P_{89} P_{12} 34567\right), \rho[2]=\left(P_{89} P_{12} 45673\right)$ and contribution $(-)^{2}$.

- (c) Now we repeat above two steps. The merging gives $\pi[3]=\left(P_{8912} 3 P_{4567}\right)$ and $\rho[3]=\left(P_{8912} P_{4567} 3\right)$, then flipping pair $\left(3 P_{8912}\right)$ gives $\pi[4]=\left(P_{8912} 3 P_{4567}\right)$ and $\rho[4]=$ $\left(P_{8912} 3 P_{4567}\right)$ with sign contribution $(-)$. Now $\pi[4]$ and $\rho[4]$ are same up to cyclic ordering and the iterating stops.

- (d) The final sign is given by $(-1)^{n-3+n_{\text {flip }}(\pi \mid \rho)}$. For our example, $n=9$ and $n_{\text {flip }}=3$, thus the overall sign is $(-)$.

Having discussed the simplest case, i.e., the CHY-integrands given by the product of two PT-factor, we move to the next simple case, i.e., all poles are at most simple poles (i.e., all subsets with index $\chi \leq 0$ ). For this situation, the integration rule has been proposed in [16]. To understand the algorithm, let us use the following example to demonstrate

$$
I=\frac{z_{12}}{z_{13} z_{16} z_{17} z_{23} z_{26}^{2} z_{27} z_{32} z_{34} z_{45} z_{51} z_{67} z_{74}} .
$$

- (a) At the first step, for the given CHY-integrand (2.1), we find all subsets $A_{i}$ having pole index $\chi\left(A_{i}\right) \geq 0 .{ }^{5}$ The case $\chi\left(A_{i}\right)=0$ corresponds the simple pole. For our example (2.9), the simple pole sets are following seven subsets:

$$
\{1,5\},\{2,3\},\{1,4,5\},\{2,3,6\},\{2,6\},\{4,5\},\{2,6,7\} .
$$

\footnotetext{
${ }^{5}$ Since from the point view of pole structures, the subset $A_{i}$ is equivalent to its complement subset $\bar{A}_{i}=\{1,2, \cdots, n\}-A_{i}$, we keep only one of them.
} 
- (b) Compatible condition: having found all poles, we need to find the compatible combination of subsets as large as possible, where each pair of subsets $\left\{A_{i}, A_{j}\right\}$ in the combination is either nested (i.e, $A_{i} \subset A_{j}$ or $A_{j} \subset A_{i}$ ) or complementary $A_{i} \bigcap A_{j}=\emptyset$. For our example (2.9), by checking (2.10) we have following six maximum combinations:

$$
\begin{aligned}
& \{\{1,5\},\{2,3\},\{1,4,5\},\{2,3,6\}\}, \quad\{\{1,5\},\{2,6\},\{1,4,5\},\{2,3,6\}\}, \\
& \{\{2,3\},\{4,5\},\{1,4,5\},\{2,3,6\}\},\{\{2,6\},\{4,5\},\{1,4,5\},\{2,3,6\}\} \text {, } \\
& \{\{1,5\},\{2,6\},\{1,4,5\},\{2,6,7\}\}, \quad\{\{2,6\},\{4,5\},\{1,4,5\},\{2,6,7\}\} \text {. }
\end{aligned}
$$

- (c) Length condition: for a maximum combinations to give a nonzero Feynman diagram, the number of subsets in the maximum combination of (2.11) should be exactly $(n-3)$. In our example, all six maximum combinations satisfy the length condition.

- (d) For each maximum compatible combination satisfying the length condition, we draw the corresponding Feynman diagram with only cubic vertices, where each propagator corresponds to a pole subset. After summing up all Feynman diagrams together, we get the final result for the starting CHY-integrand (up to a sign). For our example, it is

$$
\begin{aligned}
& \frac{1}{s_{15} s_{23} s_{145} s_{236}}+\frac{1}{s_{15} s_{26} s_{145} s_{236}}+\frac{1}{s_{23} s_{45} s_{145} s_{236}} \\
& +\frac{1}{s_{26} s_{45} s_{145} s_{236}}+\frac{1}{s_{15} s_{26} s_{145} s_{267}}+\frac{1}{s_{26} s_{45} s_{145} s_{267}}
\end{aligned}
$$

- (e) The way to determine the overall sign for the case with simple poles is a little bit complicated and more details can be found in [19, 44].

For more general CHY-integrand with higher poles, we can use the cross ratio identities [19] to reduce the degree of poles one by one until all poles are single poles, ${ }^{6}$ then we can use the integration rule reviewed above to write down analytical expressions.

\subsection{Effective Feynman diagram}

To construct one-loop CHY-integrand for a given theory, some knowledge of the corresponding Feynman diagrams will be useful. For the bi-adjoint scalar theory focused on in this paper, the concept effective Feynman diagram [3, 43], which has already appeared in the graph (d) of figure 1, becomes very important. More explicitly, for any pair of PT factors with nonzero contributions, all Feynman diagrams can be coded into one effective Feynman diagram as explained in (2.8). Another example with $[\pi \mid \rho] \equiv[12345678 \mid 12846573]$, where two orderings (up to cyclic permutations) have been separated by the vertical line, will

\footnotetext{
${ }^{6} \mathrm{~A}$ clear algorithm has been given in the appendix of [42]. Other related understanding of higher poles can be found in [41, 47].
} 
give the effective Feynman diagram in (2.13)

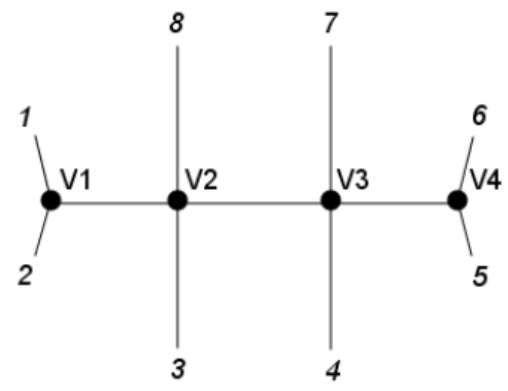

Given the effective Feynman diagram, we can infer following information:

- (1) For each effective vertex, it will split each ordering into several groups. For example, for vertex $V_{2}$ in $(2.13), \pi$-planar ordering $(\pi=[12345678])$ is split into $(12)(3)(4567)(8)$, while $\rho$-planar ordering, to $(12)(8)(4657)(3)$.

- (2) If we treat each group as an element for the splitting of a given effective vertex, the relative ordering between $\pi$ - and $\rho$-planar orderings must be either same or complete reversed. For example, for $V_{1}$, we have the $\pi$-planar ordering splitting $(1)(2)(345678) \equiv(1)(2)(P)$ and the $\rho$-planar ordering splitting $(1)(2)(846573) \equiv$ $(1)(2)(P)$, thus they are in same ordering. For $V_{2}$, we have the $\pi$-planar ordering splitting $(12)(3)(4567)(8) \equiv\left(P_{1}\right)(3)\left(P_{2}\right)(8)$ and $\rho$-planar ordering splitting $(12)(8)(4657)(3) \equiv\left(P_{1}\right)(8)\left(P_{2}\right)(3)$, thus they are in reversed ordering. For simplicity and clarity we call the former one $\mathcal{S O}$-type (abbreviation of 'same ordering') and the latter one $\mathcal{R O}$-type (abbreviation of 'reversed ordering').

- (3) Another fact is that the relative ordering of two adjacent effective vertices must be opposite. In other words, one of them is $\mathcal{S O}$-type and another is $\mathcal{R O}$-type.

- (4) These two properties can also be seen from the process of obtaining effective Feynman diagram (as explained in the figure 1). Once we fixed the planar order according to $\pi$, the order inside one polygon is determined by $\rho$, which will be either clockwise or anti-clockwise as shown in the graph A of figure 2, and two adjacent polygons are always in reversed orderings (clockwise versus anti-clockwise), as shown in the graph B of figure 2 .

Having the effective Feynman diagram drawn in the $\pi$-planar ordering, we can reach the $\rho$-planar ordering, by reversing permutation mentioned in [43]. Here we provide a pictorial manipulation over the effective Feynman diagram, which will be used for later discussion. Let us use two examples, i.e., [12345678|12846573] and [1234567|2143756], to demonstrate the idea (see figure 3 ). At the first step, we pick up an arbitrary $\mathcal{S O}$-type effective vertex. Then for each sub-part connecting to this vertex through an effective propagator, we do the axial reflection according to this propagator. After that, we move to next effective 


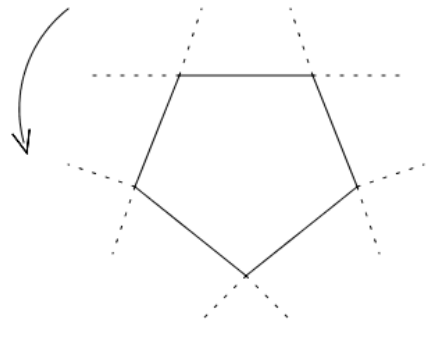

A

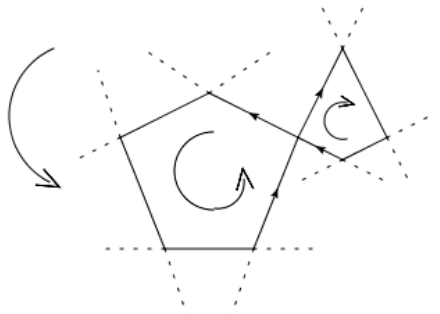

B

Figure 2. The pilot process shows two properties of effective Feynman diagram.

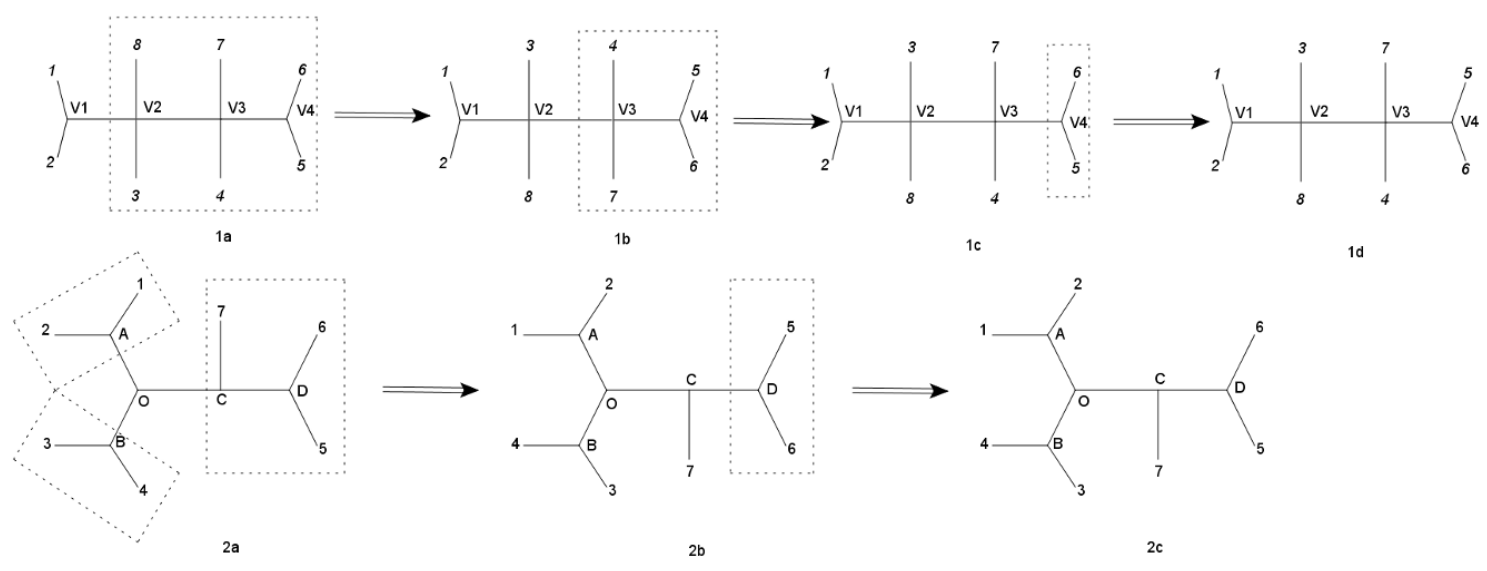

Figure 3. The process from $\pi$-ordering effective Feynman diagram to $\rho$-ordering.

vertex, and do the axial reflection to remaining parts. Repeating this procedure, finally we will end up with a $\rho$-planar ordering for the effective Feynman diagram. Let us use the upper part of figure 3 to demonstrate the algorithm. We start with $V_{1}$ vertex. Among the three parts, only the part (345678) is nontrivial under the reflection. After doing the axial reflection, we reach the graph (1b). Next, we move to the vertex $V_{2}$. Among these four parts, the three parts (3), (8), (4567) are remaining part from the point of view of $V_{1}$, thus we need to do the axial reflection for them (although for the part (3), (8) it is trivial) to reach the graph (1c). Repeating the procedure to $V_{3}$, finally we reach the graph (1d), which is in the $\rho$-planar ordering.

\section{$2.3 \quad$ Picking out poles}

In [37], to pick terms containing a particular pole structure coming from CHY-integrands, such as $(P T(1,2 \cdots))^{2}$, following cross ratio factor

$$
\mathcal{P}_{c, d}^{a, b} \equiv \frac{z_{a b} z_{c d}}{z_{a d} z_{c b}}
$$


has been introduced. For example, if we want to pick terms containing pole $s_{12}$, we do the following observation. The pole structure has split the $n$ nodes into two subsets: $A=\{1,2\}$ and $\bar{A}=\{3, \ldots, n\}$. Among these two subsets, there are two connection lines $\{2,3\}$ and $\{1, n\} .^{7}$ Using the two pairs, we can construct the cross ratio factor $\mathcal{P}_{1, n}^{2,3}$ (see (2.14)), then one can check that by multiplying this cross ratio factor, we get the CHY-integrand $(P T(1,2 \cdots))^{2} \mathcal{P}_{1, n}^{2,3}$, which will produce only these Feynman diagrams containing the pole $s_{12}$ in the original theory. Because of this role, we will call multiplying the factor $\mathcal{P}_{1, n}^{2,3}$ as "picking out the pole $s_{12}$ ".

We can pick up more than one pole at the same time. For example, to pick out poles $s_{n 12}$ and $s_{456}$ for $(P T(1,2 \cdots))^{2}$, we use the cross ratio factor $\mathcal{P}_{4,3}^{6,7}$ to pick out the pole $s_{456}$ and the cross ratio factor $\mathcal{P}_{2,3}^{n, n-1}$ to pick out the pole $s_{n 12}$. When multiplying these two together, the CHY-integrand $(P T(1,2 \cdots))^{2} \mathcal{P}_{4,3}^{6,7} \mathcal{P}_{2,3}^{n, n-1}$ does pick out all Feynman diagrams containing the pole structure $s_{456} s_{n 12}$. Similarly, the CHY-integrand $(P T(1,2 \cdots))^{2} \mathcal{P}_{1, n}^{2,3} \mathcal{P}_{3,4}^{1, n}$ does pick out all Feynman diagrams containing the pole structure $s_{12} s_{123}$.

The poles picking through some cross ratio factors has been generalized to the CHYintegrands $P T(\alpha) P T(\beta)$ in [43], where there are more connection lines between the two subsets $A$ and $\bar{A}$ since each PT-factor can give two lines. For this special case, as shown in [43], one can use either PT-factor to define the corresponding cross ratio factor, both of them give the same result. For examples, the CHY-integrand $P T(1,2,3,4,5,6) P T(1,3,4,6,5,2)$ gives $-\frac{1}{s_{12} s_{34} s_{56}}-\frac{1}{s_{12} s_{56} s_{123}}$. To pick up the pole $s_{34}$, we can use either the $P T(1,2,3,4,5,6)$ to define the cross ratio factor $\mathcal{P}_{3,2}^{4,5}$ or the $P T(1,3,4,6,5,2)$ to define the cross ratio factor $\mathcal{P}_{3,1}^{4,6}$. One can check that both of them give the same results:

$$
\begin{aligned}
& \int d \Omega_{C H Y} P T(1,2,3,4,5,6) P T(1,3,4,6,5,2) \mathcal{P}_{3,2}^{4,5}=-\frac{1}{s_{12} s_{34} s_{56}} \\
& \int d \Omega_{C H Y} P T(1,2,3,4,5,6) P T(1,3,4,6,5,2) \mathcal{P}_{3,1}^{4,6}=-\frac{1}{s_{12} s_{34} s_{56}}
\end{aligned}
$$

There are two remarks about the procedure of picking out poles. Firstly, the procedure will not change the overall sign in final result. For example, the result (2.15) has the minus sign which is the same as the one in the original theory. Secondly, as pointed out in [37, 43], there are two special cases where picking out procedure will fall: one is that there is no such a pole and another one, all terms have such a pole. For example, the CHY-integrand $P T(1,2,3,4,5) P T(1,3,2,4,5)$ gives $\frac{1}{s_{23} s_{15}}+\frac{1}{s_{23} s_{45}}$. If we naively pick out the pole $s_{34}$ from the factor $P T(1,2,3,4,5)$, we will find

$$
\int d \Omega_{C H Y} P T(1,2,3,4,5) P T(1,3,2,4,5) \mathcal{P}_{3,2}^{4,5}=\frac{1}{s_{15} s_{24}}
$$

\footnotetext{
${ }^{7}$ If there is a factor $z_{a b}$ (no matter which power) in the denominator of a given CHY-integrand, where $a \in A$ and $b \in \bar{A}$, we will call there is a connection line between the subset $A$ and its complement subset $\bar{A}$. For convenience, when we write the pair of nodes in the superscript or subscript of $\mathcal{P}$, the first is always belonging to the subset $A$ and the second one, $\bar{A}$.
} 
which is nonsense result. For the same integrand, if we try to pick out the common pole $s_{23}$ naively, we will find

$$
\int d \Omega_{C H Y} P T(1,2,3,4,5) P T(1,3,2,4,5) \mathcal{P}_{3,4}^{2,1}=\frac{1}{s_{15} s_{24}}+\frac{1}{s_{15} s_{24}}+\frac{1}{s_{13} s_{24}}+\frac{1}{s_{13} s_{45}}+\frac{1}{s_{23} s_{45}},
$$

which has additional terms comparing to the original theory. In the later part of the paper, when we apply the picking pole procedure, we need to be careful about the two special cases.

\section{One loop}

In this section, we will construct the CHY-integrands for one loop amplitudes of bi-adjoint scalar theory. The basic strategy is based on following observation: by cutting a propagator containing the loop momentum, one-loop Feynman diagram becomes the tree-level Feynman diagram. Reversing the process, i.e., by gluing two external legs of a tree-level Feynman diagram, we get an one-loop Feynman diagram, if the momenta of these two legs are massive and opposite, i.e., $k_{ \pm}= \pm L, L^{2} \neq 0$. Such a strategy has been named the "forward limit", which has been used in the literatures to construct CHY-integrands of various theories.

To see more clearly the connection of forward limit of $(n+2)$-point tree level amplitudes with two massive particles and one-loop integrand of $n$ massless particles, let us consider a typical term of one loop integrands with loop momentum $L^{8}$

$$
I\left(L, K_{1}, \ldots, K_{m}\right)=\frac{1}{L^{2}\left(L-K_{1}\right)^{2}\left(L-K_{1}-K_{2}\right)^{2} \cdots\left(L-K_{1}-\cdots-K_{m-1}\right)^{2}},
$$

where the $K_{i}$ is the $i$-th momentum attached to the loop. Applying the partial fraction identity

$$
\frac{1}{\prod D_{i}}=\sum_{i} \frac{1}{D_{i} \prod_{j \neq i}\left(D_{j}-D_{i}\right)}
$$

the (3.1) can be split to $m$ terms. For the $i$-th term with the propagator $D_{i}$, we can shift the loop momentum to $\widetilde{L} \equiv L-\sum_{t=1}^{i} K_{t}$, and the $i$-th term becomes $\frac{1}{\widetilde{L}^{2}} \frac{1}{\prod_{j \neq i}\left(D_{j}-D_{i}\right)}$. Since the loop integration is invariant under the shifting of loop momentum, we can sum them up to get a new representation of the loop integrand

$$
I\left(L, K_{1}, \cdot, K_{m}\right) \sim \frac{1}{L^{2}} \sum_{a=1}^{m} \prod_{b=a}^{a+m-2} \frac{1}{\left(L-K_{a}-K_{a+1}-\cdots-K_{b}\right)^{2}-L^{2}}
$$

where those labels are defined by module $m$. On the right hand side of (3.3), after pulling out the universal factor $\frac{1}{L^{2}}$, each remaining part can be interpreted as a ladder-like tree diagram with two external legs having on-shell massive momenta $+L$ and $-L$ momenta

\footnotetext{
${ }^{8}$ These factors do not contain the loop momentum $L$ have been omitted in (3.1).
} 
(i.e., the forward limit). With this understanding the (3.3) can be represented pictorially as

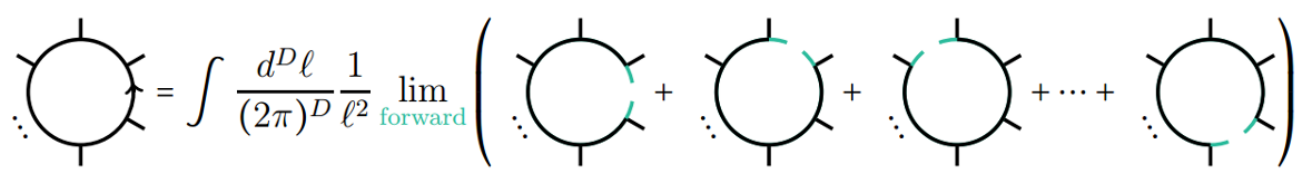

where each term inside the bracket of Right-Hand-Side of (3.4) can be obtained by cutting every loop propagator. Equation (3.4) gives the essential relation between tree-level and one-loop CHY-integrands, which will be the central object of this paper.

Based on this picture, for example in [18, 26, 27], a construction of one-loop amplitudes of bi-adjoint scalar theory by taking the forward limit of tree-level amplitudes of $n$ massless and two massive particles (or two massless particle in higher dimension) has been proposed. For general cases the expression

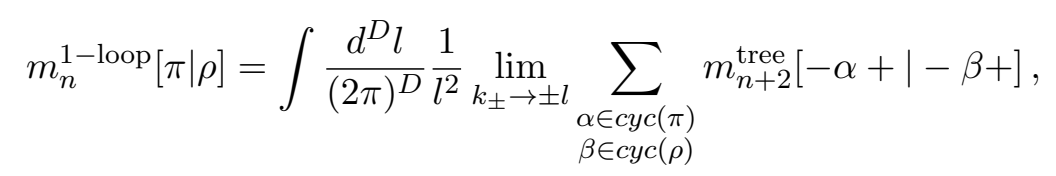

has been given in [26], where

$$
m_{n+2}^{\text {tree }}[-\alpha+\mid-\beta+]=\int d \Omega_{C H Y} P T(-, \alpha(1), \cdots, \alpha(n),+) P T(-, \beta(1), \cdots, \beta(n),+)
$$

is the tree-level amplitudes of $(n+2)$ particles defined by the CHY-integrand of two PT factors. In (3.6), +, - denote two extra massive particles which can be treated as loop momentum after taking forward limit.

Now we discuss some technical and subtle details. The pictorial representation given in (3.4) has, in fact, not one-to-one correspondence. The standard one-loop Feynman diagrams at the 1.h.s. of (3.4) should be the connected and amputated diagrams while the r.h.s. contains, in the principle, tadpoles and massless bubbles after gluing. ${ }^{9}$ These tadpole and massless bubble diagrams are singular under the forward limit, thus one needs to deal with them carefully under the limit. As shown in [26], we should pay attention to the relative ordering between following two processes: solving the tree-level scattering equations and taking the forward limit. According to the different orderings, we can divide them to following three limits:

- (A) If we solve the tree-level scattering equations first, we will have $(n-1)$ ! solutions. Among these solutions, $(n-1) !-2(n-2)$ !'s are regular, while $(n-2)$ !'s are Singular I and another $(n-2)$ !'s are Singular II. After summing over all solutions for a given CHY-integrand, we take the forward limit $k_{ \pm} \rightarrow \pm L$.

- (B) Without gauge fixing the scattering equations of $k_{ \pm}$, we take the forward limit over the tree-level scattering equations first. Then we solve the resulted one-loop scattering equations. We will have $(n-1) !-(n-2)$ ! solutions, which include the regular one and Singular I. Finally we sum them over for a given CHY-integrand.

\footnotetext{
${ }^{9}$ In the formula (3.5), tadpoles have been removed, but the massless bubbles are left.
} 
- (C) After gauge fixing the scattering equations of $k_{ \pm}$, we take the forward limit over the tree-level scattering equations first. Then we solve the resulted one-loop scattering equations. We will have $(n-1) !-2(n-2)$ ! solutions, which include only the regular one. Finally we sum them over for a given CHY-integrand.

It is clear that if the CHY-integrands behave good enough, i.e., singular solutions give zero contributions, the difference among these three categories, i.e., the contributions of Singular I and Singular II solutions are all same. But in general, singular solutions do give nonzero contributions, thus three different orderings of limits will lead to different expressions. However, different expressions do not necessarily mean contradiction in the theory. As emphasized in [27], we should treat loop integrands as "equivalent classes" modulo integrals that are scaleless (thus their loop integrations are zero). In fact, [27] pointed out that as long as CHY-integrand is homogeneous in loop momentum $L$ under the forward limit, singular solutions contribute only to scaleless terms, thus choosing the Limit (C) provides a natural regularization. The theory we are focusing in this paper, i.e., the bi-adjoint scalar theory, belongs to the homogeneous category discussed in [27], thus using only regular solutions should be a good practice, especially doing the numerical calculations. However, if we want to find the analytic expression, as carefully analyzed in [27], summing only over regular solutions will produce unphysical poles. Unlike the treelevel case, we have various ways (such as the integration rule reviewed in previous section) to write down analytic expressions. To surround this difficulty, in this paper we will take the different approach, i.e., using the Limit (A).

Our new approach, i.e., using the Limit (A), has its advantage and disadvantage when including the singular solutions. On the one hand, since we have summed over all solutions, methods like the integration rule can be applied directly. On the other hand, diagrams like tadpoles and massless bubble will cause singular behavior under the forward limits, thus we need to treat them carefully. Our treatment is to subtract these singular terms directly from the CHY-integrands (3.5) by using the picking out poles procedure reviewed in the previous section.

Having discussed the idea, the structure of this section is following. In the first subsection, we discuss the case where two orderings $\pi, \rho$ are same. In the second subsection, we discuss the case where two orderings $\pi, \rho$ are opposite to each other. In the third subsection, we discuss the general case. In the fourth subsection, we present some examples.

\section{$3.1 \pi=\rho$}

In this subsection, we will focus on the special case $\pi=\rho$, so $m_{n}^{1-\text { loop }}[\pi \mid \pi]$ will be the sum of all possible $n$-point amputated one loop Feynman diagrams with the $\pi$-planar ordering. Without loss of generality, we can choose $\pi=(1,2, \cdots, n)$.

To get some sense for possible one-loop CHY-integrand, we need to capture relations between one-loop diagrams and tree diagrams. These relations can be seen by two types of processes as follows. Recalling the expansion (3.4), we see terms in the r.h.s. of (3.4) are the ladder-like $(n+2)$-point tree diagrams obtained by cutting each propagator of loop circle in the l.h.s. of (3.4) (we call it "cutting" process). With the ordering of external 


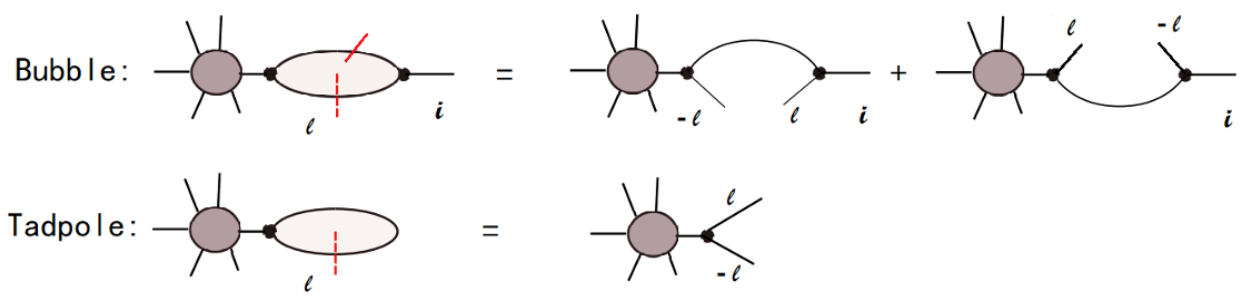

Figure 4. The should-be-excluded one-loop Feynman diagrams of $\phi^{3}$ theory and their corresponding tree diagrams after cutting.

particles as $(1,2, \cdots, n)$, we could see the orderings of particles in those tree diagrams is $(1,2, \cdots, i,+,-, i+1, \cdots, n)$, which is obtained by inserting the pair $(+,-)$ into two adjacent legs $(i, i+1)$ with $i=1,2, \ldots, n$. On the other hand, given an arbitrary $(n+$ $2)$-point planar tree diagram with the planar ordering $(1,2, \cdots, i,+,-, i+1, \cdots, n)$, we can obtain an one-loop diagram with the planar ordering $(1,2, \cdots, n)$ by linking the two external legs + and - (we denote this procedure as "gluing" process).

With above cutting and gluing processes, one natural question is that after cutting process will two different one-loop diagrams produce a same $(n+2)$-point planar tree diagram? Apparently the answer is no, because by the gluing process, a $(n+2)$-point planar tree diagram will produce one and only one one-loop diagram. This argument shows that the cutting process and the gluing process, are inverse operation to each other. Thus we have the identity:

$$
\begin{aligned}
& \widetilde{M}_{n}^{1-\operatorname{loop}}[12 \cdots n] \\
& \quad=\int \frac{d^{D} l}{(2 \pi)^{D}} \frac{1}{l^{2}} \lim \sum_{1 \leq i \leq n} m_{n+2}^{\text {tree }}[1,2, \cdots, i,+,-, i+1, \cdots, n \mid 1,2, \cdots, i,+,-, i+1, \cdots, n]
\end{aligned}
$$

where we have used the notation $\widetilde{M}_{n}^{1-\text { loop }}[12 \cdots n]$ instead of $m_{n}^{1-\text { loop }}[12 \cdots n]$. The reason is that when gluing tree diagrams at the r.h.s. of (3.7), it will produce the one-loop tadpole and massless bubble diagrams (see figure 4 ), which should not be included for the standard one-loop amplitude $m_{n}^{1-\text { loop }}[12 \cdots n]$.

Now it is clear that to obtain the wanted one-loop amplitude $m_{n}^{1-\text { loop }}[12 \cdots n]$, we need to subtract tree diagrams from the r.h.s. of (3.7), which will produce tadpole and massless bubble diagrams after gluing process. These tree diagrams have special pole structures. From the figure 4 , we see that if the $(n+2)$-point tree diagram contains the pole $s_{+-}$, it will produce the tadpole, while if it contains the pole $s_{i+} s_{i+-}$ or $s_{-i} s_{+-i}$, it will produce the massless bubble of the leg $i$. Knowing the special pole structures, the procedure of 'picking out poles' reviewed in the previous section come to play. To isolate tree diagrams contributing to tadpoles, we multiply the factor $\mathcal{P}_{-, i+1}^{+, i}$ to the CHY-integrand $(P T(1,2, \ldots, i,+,-, i+1, \ldots, n))^{2}$. To isolate tree diagrams contributing to massless bubbles, we multiply the factor $\mathcal{P}_{+,-}^{i, i-1} \mathcal{P}_{-, i+1}^{i, i-1}$ to the CHY-integrand $(P T(1,2, \ldots, i,+,-, i+$ $1, \ldots, n))^{2}$ for massless bubble on the leg $i$ and the factor $\mathcal{P}_{i+1, i+2}^{-,+} \mathcal{P}_{i+1, i+2}^{+, i}$ to the CHY- 
integrand $(P T(1,2, \ldots, i,+,-, i+1, \ldots, n))^{2}$ for massless bubble on the leg $(i+1)$. Subtracting them, we arrive the following one loop CHY-integrand ${ }^{10}$

$$
\begin{aligned}
m_{n}^{1-\text { loop }} & {[1,2, \cdots, n \mid 1,2, \cdots, n] } \\
= & \int \frac{d^{D} l}{(2 \pi)^{D}} \frac{1}{l^{2}} \lim _{k \pm \biguplus \pm l} \int d \Omega_{C H Y} \sum_{i=1}^{n}(P T(1,2, \cdots, i,+,-, i+1, \cdots, n))^{2} \\
& \times\left\{1-\mathcal{P}_{-, i+1}^{+, i}-\mathcal{P}_{+,-}^{i, i-1} \mathcal{P}_{-, i+1}^{i, i-1}-\mathcal{P}_{i+1, i+2}^{-,+} \mathcal{P}_{i+1, i+2}^{+, i}\right\}
\end{aligned}
$$

with all labels in $\mathcal{P}$ are understood as $\bmod n$.

Now we present an example to demonstrate our result (3.8) and compare it with the one given by (3.5). For $n=4$, a typical term after cutting is the tree-level Feynman amplitude $m_{6}^{\text {tree }}[1,2,3,4,+,-\mid 1,2,3,4,+,-]$. It contains following 14 diagrams

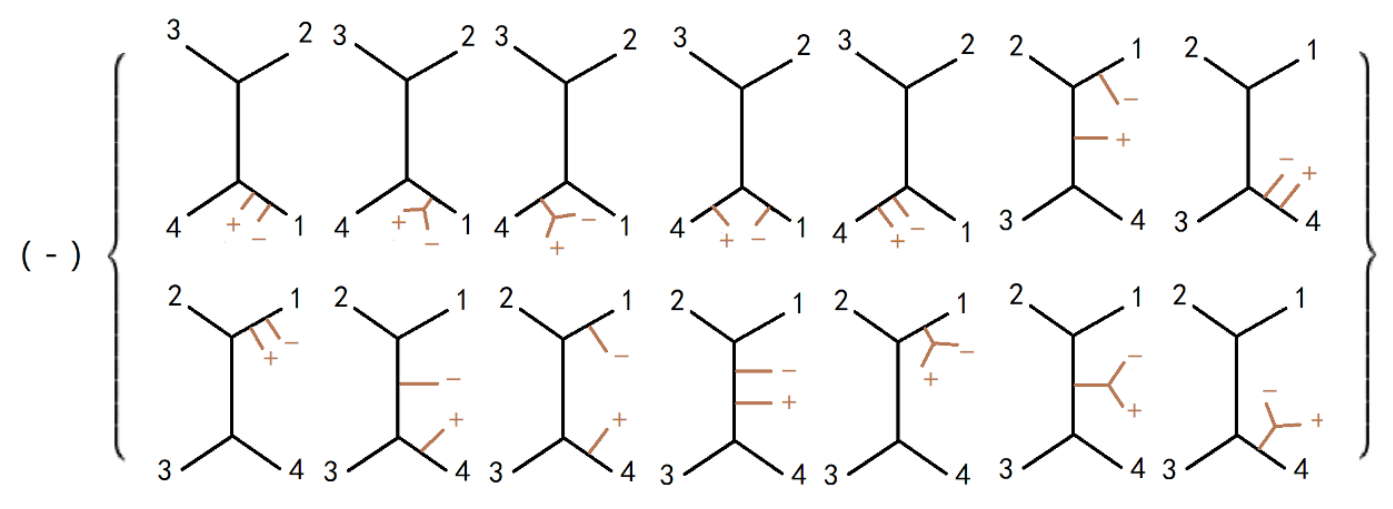

Among these 14 diagrams, five contain the pole $s_{+-}$and it is easy to check by explicit calculation that

$$
\int d \Omega_{C H Y} \mathcal{P}_{-, 1}^{+, 4}(\operatorname{PT}(1,2,3,4,+,-))^{2}
$$
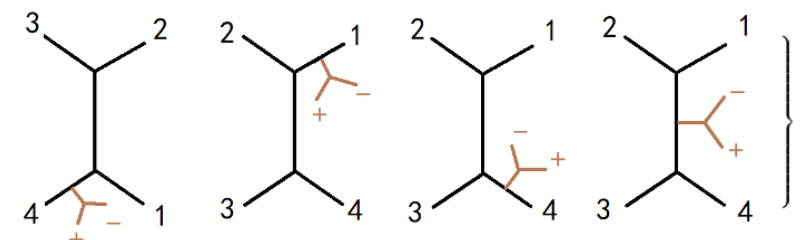

\footnotetext{
${ }^{10}$ In [18], CHY-integrand for the case $\pi=\rho$ has also been constructed, where the tadpoles and massless bubbles have not been subtracted. However, with the gauge fixing of $z_{+}, z_{-}$to $0, \infty$ and the proper regularization, these singular contributions have been controlled.
} 
Similarly, one can check

$$
\int d \Omega_{C H Y} \mathcal{P}_{+,-}^{4,3} \mathcal{P}_{-, 1}^{4,3}(\operatorname{PT}(1,2,3,4,+,-))^{2}
$$

picking out all diagrams containing poles $s_{4+-} s_{4+}$, and

$$
\int d \Omega_{C H Y} \mathcal{P}_{1,2}^{-,+} \mathcal{P}_{1,2}^{+, 4}(P T(1,2,3,4,+,-))^{2}
$$

picking out all diagrams containing poles $s_{+-1} s_{-1}$. Subtracting these singular contributions we get:

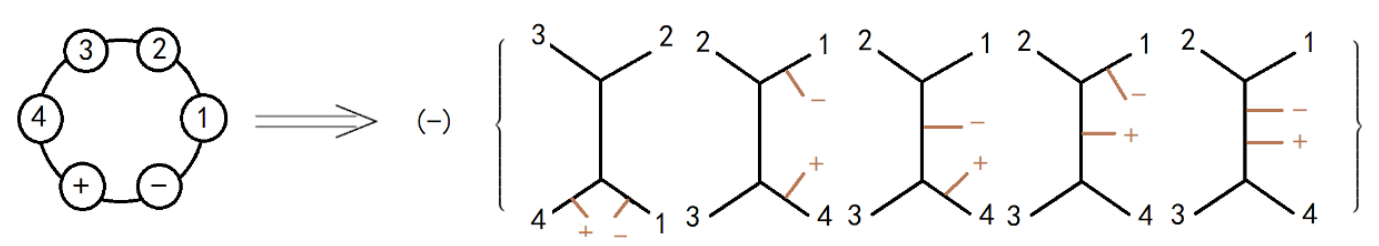

For other three terms, we just need to cyclically permutate $(1,2,3,4)$ and obtain:

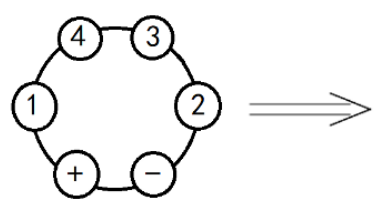<smiles>CC(C)C(C)C</smiles><smiles>CC(C)C(C)C(C)I</smiles><smiles>[1H]C(I)C([3H])C([2H])C</smiles><smiles>CC(I)C(C)C(C)I</smiles><smiles>[2H]C(C)C(C)C(C)I</smiles><smiles>C1=CC2OCOCOB(O1)O2</smiles>
$(-)$<smiles>CC(I)C1CC1</smiles><smiles>CC(C)C(C)C(C)I</smiles><smiles>[2H]C(I)C(C)C(C)C</smiles><smiles>CC(I)C(C)C(C)I</smiles><smiles>[2H]C(I)C(C)C(C)C</smiles> 

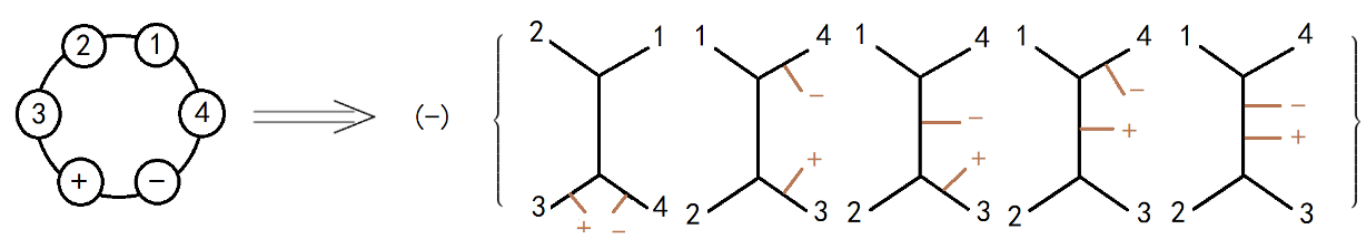

(3.16)

When we glue all,+- legs as required by the forward limit, we reach following one loop diagrams:

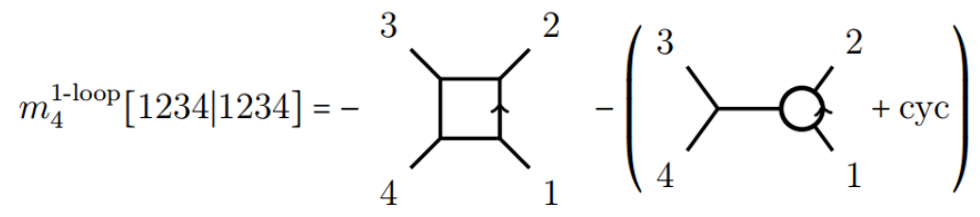

$$
\begin{aligned}
& -\left({ }_{4}^{3}-\varkappa_{1}^{2}+\text { cyc }\right)
\end{aligned}
$$

Thus we have presented four point example for (3.8). Compared with (3.5), the difference is:

$$
\Delta_{4} m_{4}^{\text {tree }}[1234 \mid 1234] \equiv-\frac{1}{4} \int \frac{d^{D} l}{(2 \pi)^{D}} \frac{1}{l^{2}} \sum_{a=1}^{n} \frac{1}{\left(l \cdot k_{a}\right)^{2}} \times m_{4}^{\text {tree }}[1234 \mid 1234]
$$

which follows from

$$
\lim _{k_{ \pm} \rightarrow \pm \ell}\left(\Upsilon^{a} a+\bigcirc a\right)=-\frac{1}{4\left(l \cdot k_{a}\right)^{2}} \times \bigcirc-a, \quad \forall a
$$

Thus the difference between (3.5) and (3.8) is exactly those tree diagrams which contribute to massless bubbles. Since its scale-free from, the integration of (3.17) is zero. Thus these two results are in the same equivalent class. Compared with (3.5) with $n^{2}$ terms, for the special case $\pi=\rho$, we have only $4 n$ terms. Furthermore, although the r.h.s. of (3.19) is regular, each term at the l.h.s. is singular, thus when we take the forward limit.

\section{$3.2 \pi=\rho^{T}$}

Now we consider another special case, i.e., the two orderings are opposite $\pi=\rho^{T}$, where $T$ means the reversing ordering of the $\rho$. If it is for tree level amplitude, since $m_{n}^{\text {tree }}[\pi \mid \rho]=$ $(-)^{n} m_{n}^{\text {tree }}\left[\pi \mid \rho^{T}\right]$, we will have $m_{n}^{\text {tree }}\left[\pi \mid \pi^{T}\right]=(-)^{n} m_{n}^{\text {tree }}[\pi \mid \pi]$. However, the situation is different for one loop case. As shown in [26], $m_{4}^{1-\text { loop }}[1234 \mid 4321]$ contains only two oneloop diagrams, while $m_{4}^{1-\text { loop }}[1234 \mid 1234]$ contains box, triangle and bubble diagrams as given in (3.17). The reason is that when we go from the ordering $\pi$ to the ordering $\rho$, we need to flip legs at some cubic vertexes. However, such flips are not allowed if the leg 

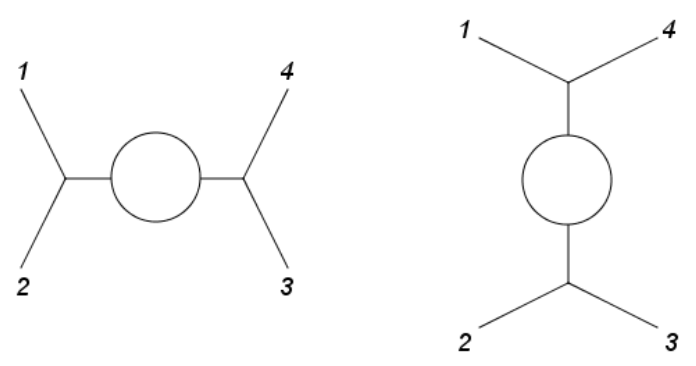

Figure 5. $m_{4}^{1-\operatorname{loop}}[1234 \mid 4321]$.

containing the loop momentum. Thus in this case, it is easy to see that all allowed oneloop diagrams are massive bubble diagrams. The reason is following. If a loop diagram is compatible with the $\pi$ ordering, then the $\pi$ ordering can be separated to $m$ groups $\left\{\Pi_{1}\left|\Pi_{2}\right| \ldots \mid \Pi_{m}\right\}$, where each group is attached to the loop at a cubic vertex. When we do the flipping, we are allowed to flip only inside each group, thus we can produce these orderings $\left\{\mathcal{F}\left[\Pi_{1}\right]\left|\mathcal{F}\left[\Pi_{2}\right]\right| \ldots \mid \mathcal{F}\left[\Pi_{m}\right]\right\}$. Then if $m \geq 3$, it is impossible to go from $\pi$-ordering to $\pi^{T}$-ordering. When $m=2$, it is easy to see that

$$
\pi \rightarrow\left\{\Pi_{1} \mid \Pi_{2}\right\} \rightarrow\left\{\Pi_{1}^{T} \mid \Pi_{2}^{T}\right\}=\left\{\Pi_{2}^{T} \mid \Pi_{1}^{T}\right\}=\pi^{T} .
$$

Furthermore, starting from the tree level diagrams, we can insert the loop to each internal propagator. Collecting all of them, we get the $m_{n}^{1-\text { loop }}\left[\pi \mid \pi^{T}\right]$.

Now we discuss how to construct the one-loop CHY-integrand by above understanding. We choose $\pi=(1,2, \cdots, n), \rho=(n, \cdots, 2,1)$ without loss of generality. Let us consider a typical diagram of the amplitude $M_{n}^{1-\text { loop }}[1,2, \cdots, n \mid n, \cdots, 2,1]$ drawing with the clockwise ordering $(1,2, \cdots, n)$ :

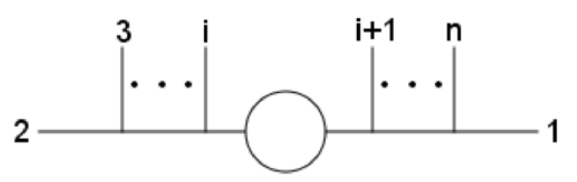

where $i$ can be any integer of $3 \leq i \leq n-1$. After flipping, from the diagram (3.21) we can produce following diagram with the $(n, \cdots, 2,1)$-planar ordering

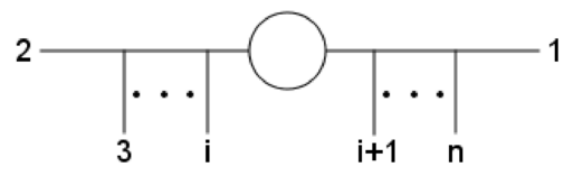


Cutting each loop propagator in (3.21), we get two $(n+2)$-point tree diagrams as following
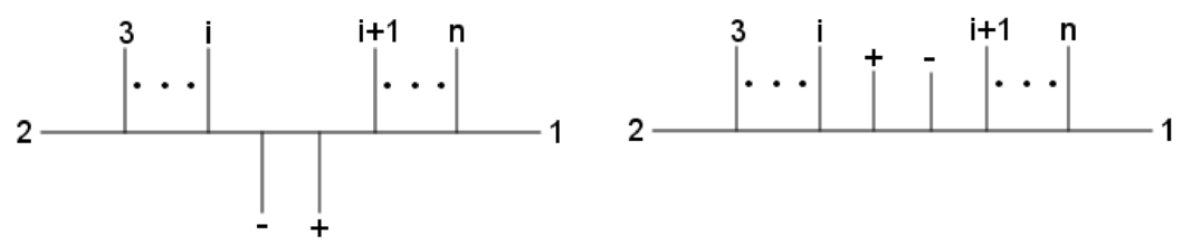

while cutting the corresponding loop diagram (3.22) becomes
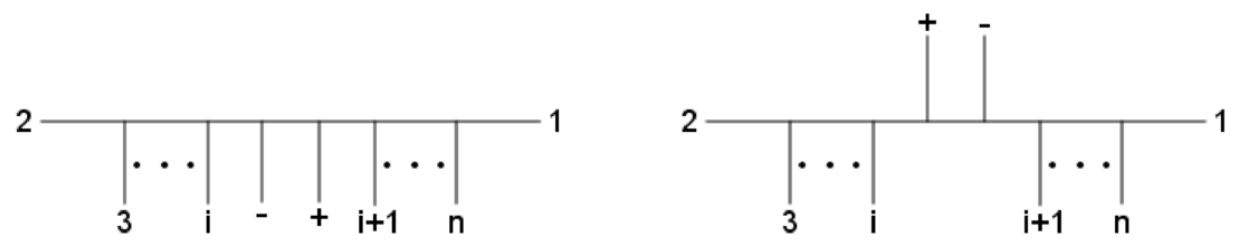

An important point is that among these two diagrams (3.23) and (3.24), there is a oneto-one correspondence relation: the left one in (3.23) corresponds to the left one in (3.24) and similarly the right one to the right one. From the correspondence, we can see that the left comes form the tree level CHY-integrand

$$
P T(1,+,-, 2, \cdots, n) P T(n, \cdots, i+1,+,-, i, \cdots, 1), \quad 3 \leq i \leq n-1 .
$$

It is easy to check that the CHY-integrand (3.25) produces terms containing tadpole $s_{+-}$, but not the massless bubbles. Thus using the operator $\mathcal{P}_{-, 2}^{+, 1}$, we can pick up tadpole contributions.

In above discussion, we have required $3 \leq i \leq n-1$. Now we consider the case $i=2$ and $i=n$. For these cases, the corresponding CHY-integrands are given by

$$
\begin{aligned}
& P T(1,+,-, 2, \cdots, n) P T(n, \cdots,+,-, 2,1) \\
& P T(1,+,-, 2, \cdots, n) P T(n, \cdots, 2,1,+,-)
\end{aligned}
$$

and their effective Feynman diagrams are following respectively
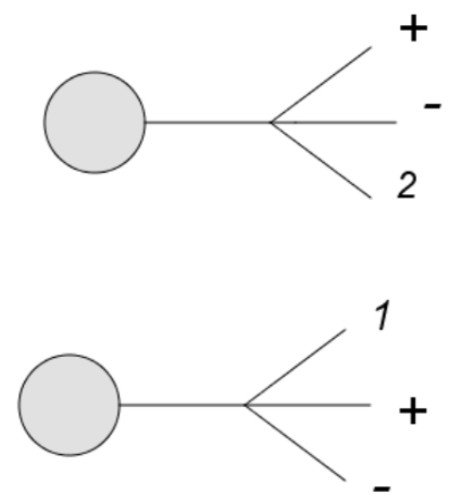
All these diagrams in (3.28) and (3.29) contain either massless bubbles or tadpoles, thus we should not consider these terms in the CHY-integrand. Now for a given insertion of ,+- legs in $\pi$-ordering, i.e., the fixed factor $P T(1,+,-, 2, \cdots, n)$, we have considered all possible insertion in the $\pi^{T}$-ordering, except the following one $\operatorname{PT}(n, \cdots, 2,+,-, 1)$. It is easy to see that

$$
P T(1,+,-, 2, \cdots, n) P T(n, \cdots, 2,+,-, 1)=\mathcal{P}_{-, 1}^{+, 2} P T(1,+,-, 2, \cdots, n) P T(n, \cdots, 2,-,+, 1)
$$

which means all Feynman diagrams comings from (3.30) have pole structure $s_{+-}$. In other words, it gives only tadpole diagrams, so we should drop this term.

Above discussion tells us what will happen when one insert,+- legs between $\{1,2\}$ in the $\pi$-ordering. Similar result can be obtained for the insertion between another pair $\{i,(i+1)\}$. When we sum up all these pairs together, we arrive following proposal for one loop CHY-integrand of $m^{1-\operatorname{loop}}\left[\pi \mid \pi^{T}\right]$

$$
\begin{aligned}
m_{n}^{1-\text { loop }} & {[1,2, \cdots, n \mid n, \cdots, 2,1] } \\
= & \int \frac{d^{D} l}{(2 \pi)^{D}} \frac{1}{l^{2}} \lim _{k_{ \pm} \mapsto \pm l} \int d \Omega_{C H Y} \\
& \times \sum_{i, j \neq i, i \pm 1}\left(1-\mathcal{P}_{-, i+1}^{+, i}\right) \operatorname{PT}(\cdots, i,+,-, i+1, \cdots) P T(\cdots j+1,+,-, j, \cdots) .
\end{aligned}
$$

where the factor $\left(1-\mathcal{P}_{-, i+1}^{+, i}\right)$ removes the tadpole contribution effectively.

Now we present an example, i.e., $\pi=(1234)$ and $\rho=(4321)$. Before taking the forward limit, the r.h.s. of (3.31) gives following four terms

$$
\begin{aligned}
& \left(1-\mathcal{P}_{-, 2}^{+, 1}\right) P T(1,+,-, 2,3,4) P T(4,+,-, 3,2,1) \\
& +\left(1-\mathcal{P}_{-, 3}^{+, 2}\right) \operatorname{PT}(1,2,+,-, 3,4) P T(4,3,2,1,+,-) \\
& +\left(1-\mathcal{P}_{-, 4}^{+, 3}\right) \operatorname{PT}(1,2,3,+,-, 4) P T(4,3,2,+,-, 1) \\
& +\left(1-\mathcal{P}_{-, 1}^{+, 4}\right) \operatorname{PT}(1,2,3,4,+,-) P T(4,3,+,-, 2,1) .
\end{aligned}
$$

Each term gives one diagram in following result
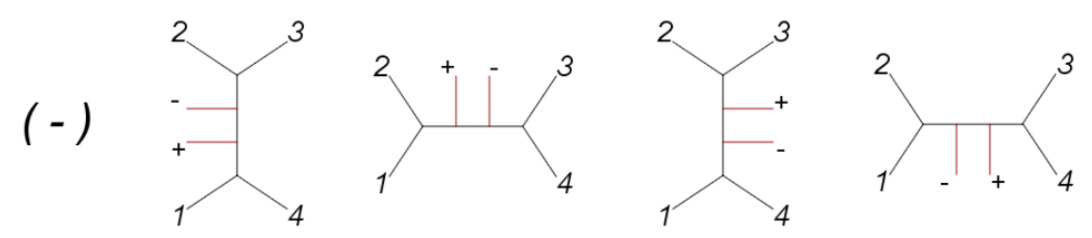

Since there is no any singularity left, one can take the forward limit and obtain

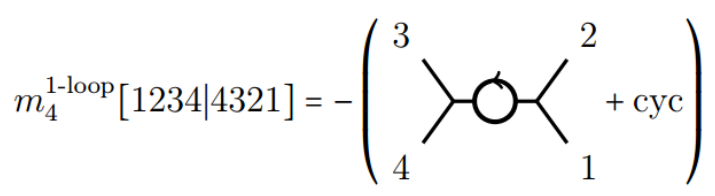



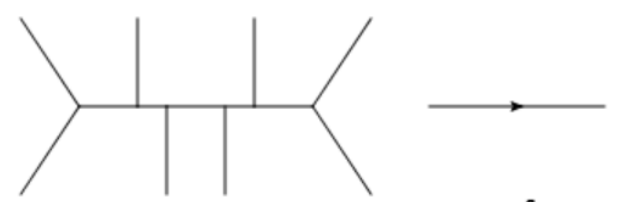

$A$

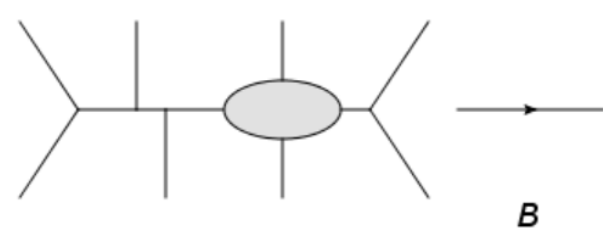

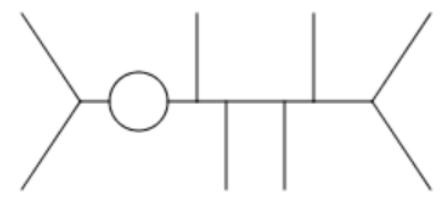

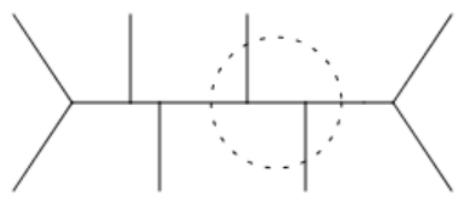

Figure 6. (A) The way constructing one-loop diagram from tree. (B) The way constructing tree diagram from one-loop diagram.

Compared with the proposal (3.5), again we do not have following massless bubble terms

$$
\Delta_{4} m_{4}^{\text {tree }}[1234 \mid 4321] \equiv-\frac{1}{4} \int \frac{d^{D} l}{(2 \pi)^{D}} \frac{1}{l^{2}} \sum_{a=1}^{n} \frac{1}{\left(l \cdot k_{a}\right)^{2}} \times m_{4}^{\text {tree }}[1234 \mid 4321]
$$

\subsection{For general orderings $\pi$ and $\rho$}

Having dealt with two special cases, now we move to general cases, i.e., with arbitrary orderings of $\pi$ and $\rho$. The first important observation is that $m_{n}^{1-\text { loop }}[\pi \mid \rho] \neq 0$ if and only if $m_{n}^{\text {tree }}[\pi \mid \rho] \neq 0$. The argument is follows. If $m_{n}^{\text {tree }}[\pi \mid \rho] \neq 0$, we can obtain oneloop diagrams respecting both $\pi$ - and $\rho$-planar orderings by adding an bubble on any internal propagator. On the other hand, given any one-loop diagram in $m_{n}^{1-\text { loop }}[\pi \mid \rho]$, we can construct the corresponding tree diagrams respecting both $\pi$ - and $\rho$-planar orderings. The procedure is follows. At the first step, we shrink the loop to a point, so we get an effective vertex. Then we can expand the effective vertex to any tree diagram with only cubic vertexes. Above argument is illustrated in the figure 6 .

With above observation, now we show how to construct the one-loop CHY-integrand from the corresponding tree-level effective Feynman diagram. For clarity, we will use the example $\pi=(1,2,3,4,5,6,7,8)$ and $\rho=(1,2,8,4,6,5,7,3)$ to demonstrate our construction.

At the first step, we insert a loop to the effective tree level Feynman diagram. A key point is that after the insertion of the loop, no any effective propagator should be replaced. Let us see what will happen if an effective propagator has been replace. For example, we have drawn three graphs in the figure 7 according to the planar ordering $\pi$ of above example, where the loop in the graphs $A, B$ has replaced the effective propagator $s_{1238}, s_{56}$ respectively while the loop in the graphs $C$ has replaced two effective propagators $s_{1238}, s_{12}$. It is easy to see that by flips, we can not transform the planar ordering $\pi$ to the ordering $\rho$ (remembering that we can not flip lines containing loop momentum). The reason is simple. As we have shown before, two effective vertexes connecting by a given effective propagator must have opposite ordering, i.e., one is the $\mathcal{S O}$-type and another one, $\mathcal{R O}$-type. Thus to 


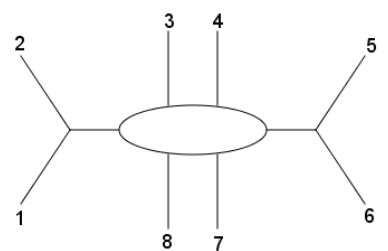

A

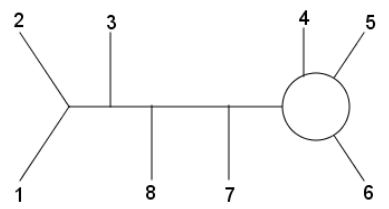

B

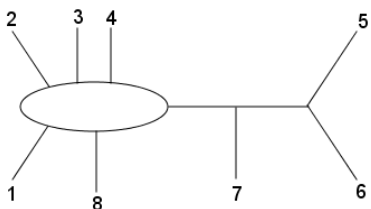

Figure 7. Some one loop Feynman diagrams cover more than one effective vertexes.

transform the $\pi$-ordering to the $\rho$-ordering, we need to flip at the vertex having reversing ordering. However, if such an effective propagator has been replaced by the loop, the needed flips do not exist, thus at the loop level, we can not transform $\pi$-ordering to $\rho$-ordering.

Above clarification shows that there are two types of insertions of the loop. The first type is to inserted on the effective propagators as well as on the external lines ${ }^{11}$
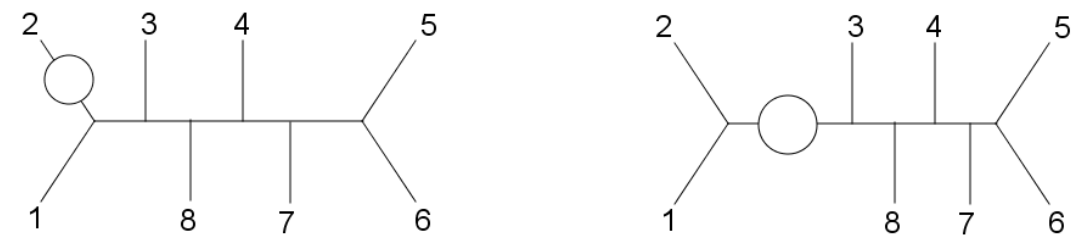

The second type is rest cases, where the loop is not inserted either on the effective propagators or on the external lines as shown in following:
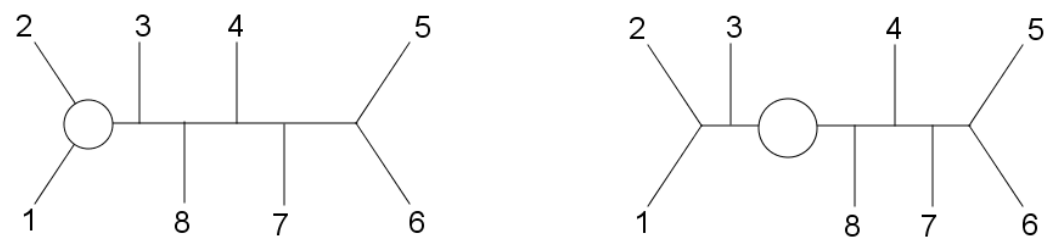

Having classified the type of loops, we can attribute the inserted loops to the corresponding effective vertexes. For the second type of loops, it must belong to a particular effective vertex. For the first type of loops, there are two cases. If it is inserted on the effective propagator, it can be attribute to each of effective vertexes at the two ends of the

\footnotetext{
${ }^{11}$ Here we have included the massless bubbles when considering the construction of CHY-integrands. Later we will move these singularities away.
} 
propagator. If it is inserted on the external legs, it must belong to a particular effective vertex. With such an assignment, all loop Feynman diagrams connecting to an effective vertex $V_{i}$ can be collected together and denoted as $\widehat{m}_{\mathcal{S O} / \mathcal{R O}}^{1-\text { loop }}\left[V_{i}\right]$ where $\mathcal{S O}, \mathcal{R O}$ mean the $\mathcal{S O}, \mathcal{R O}$ type vertexes respectively. For instance, $\widehat{m}_{\mathcal{S O}}^{1-\text { loop }}\left[V_{1}\right]$ and $\widehat{m}_{\mathcal{R O}}^{1-\text { loop }}\left[V_{2}\right]$ in our example are shown as follows.
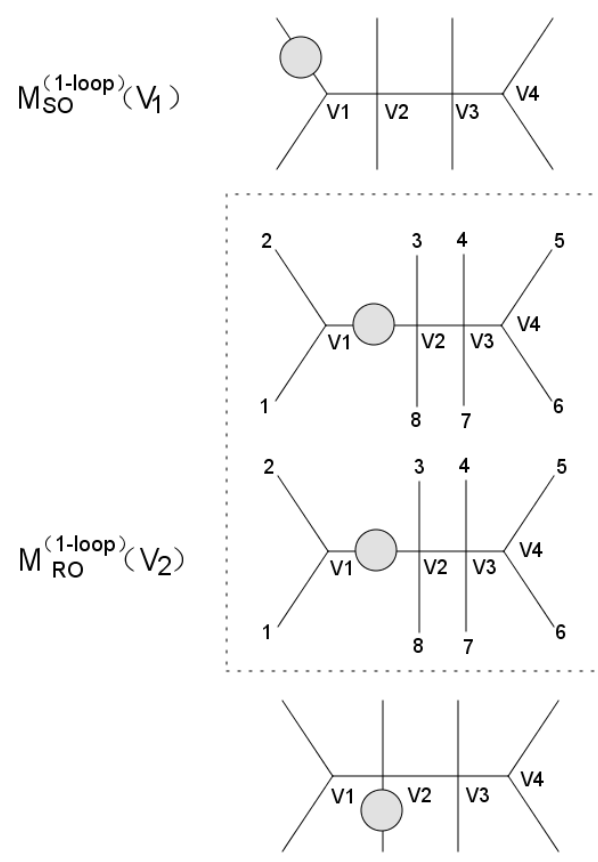
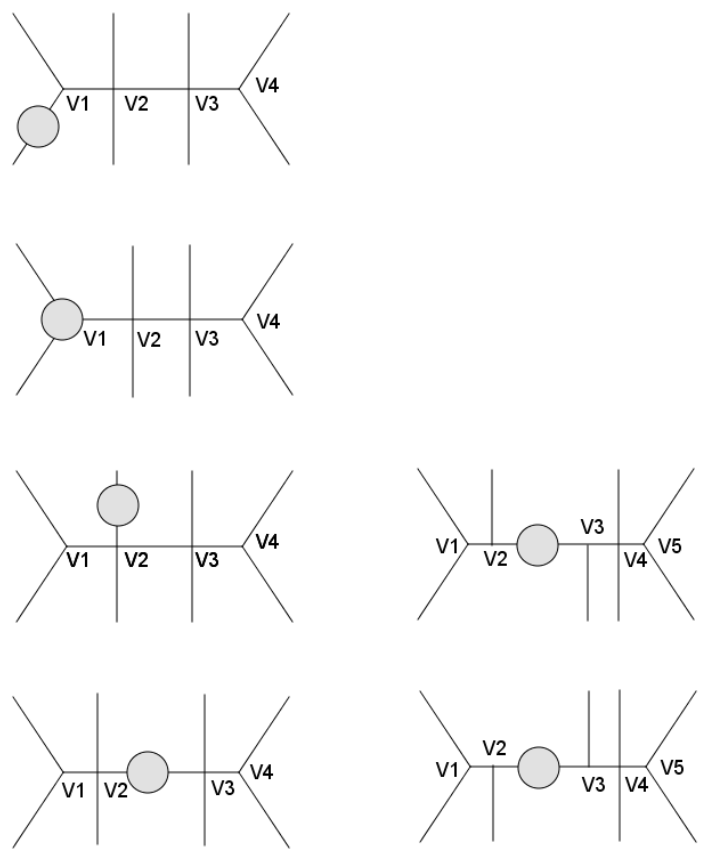

With such a grouping, we can write

$$
\widehat{m}_{n}^{1-\text { loop }}[\pi \mid \rho]=\bigcup_{V_{i}} \widehat{m}_{\mathcal{S O} / \mathcal{R O O}}^{1-\text { loop }}\left[V_{i}\right]
$$

where we use union instead of sum because as we have mentioned before, the loop inserted on the effective propagator has been assigned to two effective vertexes as shown in (3.38) by the dashed box. Now the problem is reduced to find out the CHY-integrands for each $\widehat{m}_{S O / R O}^{1-\text { loop }}\left[V_{i}\right]$ 's.

For an arbitrary effective vertex $V_{i}$ as

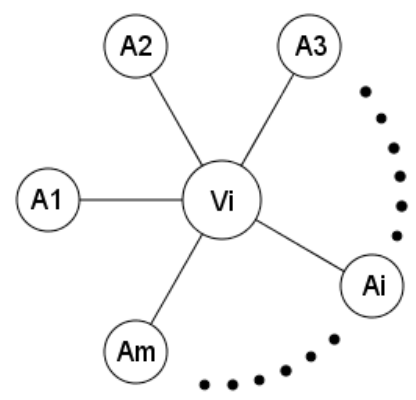


if it is the $\mathcal{S O}$-type, the $\pi$ - and $\rho$-planar ordering splitting must be the form

$$
\begin{aligned}
\pi-\text { split } & =\left(A_{1}\right)\left(A_{2}\right) \cdots\left(A_{m}\right) \\
\rho-\text { split } & =\left(A_{1}^{\prime}\right)\left(A_{2}^{\prime}\right) \cdots\left(A_{m}^{\prime}\right)
\end{aligned}
$$

where $A_{i}^{\prime}$ is a permutation ${ }^{12}$ of $A_{i}$. If it is the $\mathcal{R O}$-type, the splitting must be

$$
\begin{aligned}
\pi-\text { split } & =\left(A_{1}\right)\left(A_{2}\right) \cdots\left(A_{m}\right) \\
\rho^{\prime}-\text { split } & =\left(A_{m}^{\prime}\right) \cdots\left(A_{2}^{\prime}\right)\left(A_{1}^{\prime}\right)
\end{aligned}
$$

When all $A_{i}$ in (3.41) and (3.42) contain only one external leg, it is just reduced to the two special cases $\widehat{m}_{n}^{1-\text { loop }}[1,2, \cdots, n \mid 1,2, \cdots, n]$ or $\widehat{m}_{n}^{1-\text { loop }}[1,2, \cdots, n \mid n, \cdots, 2,1]$ studied in previous subsections. Now it is clear how to extend the construction to general cases: we treat each group $A_{i}$ as a single element and insert $(+,-)$ between each neighboring group in both orderings at same time. More explicitly, in the case (3.41), we can construct formally as follows:

$$
\widehat{m}_{S O}^{1-\text { loop }}[V]=\sum_{i}\left(1-\mathcal{P}_{-, \cdot}^{+, \cdot}\right) \operatorname{PT}\left(\cdots\left(A_{i}\right)+-\left(A_{i+1}\right) \cdots\right) P T\left(\cdots\left(A_{i}^{\prime}\right)+-\left(A_{i+1}^{\prime}\right) \cdots\right)
$$

where $\cdot$ in $\mathcal{P}$ denote the right-most particle next to $+l$ and left-most particle next to $-l$. While in the case (3.42), we have formally

$$
\widehat{m}_{R O}^{1-\text { loop }}[V] \subseteq \sum_{i=1}^{m}\left\{\left(1-\mathcal{P}_{-, \cdot}^{+, \cdot}\right) \sum_{j \neq i} P T\left(\cdots\left(A_{i}\right),+,-,\left(A_{i+1}\right), \cdots\right) P T\left(\cdots\left(A_{j+1}^{\prime}\right),+,-,\left(A_{j}^{\prime}\right) \cdots\right)\right\} .
$$

In (3.43) and (3.44), the combination $\left(1-\mathcal{P}_{-, \cdot}^{+, \cdot}\right)$ has been used to eliminate the tadpole contributions. Furthermore, in (3.44) the summation region is larger than the one in (3.31) where $j \neq i, i \pm 1$. The reason is follows. An external line of an effective vertex could be the effective propagator, thus the inserted loop on this line should be kept. Because the same reason, in (3.43) we have not removed the external bubbles.

Another important point in (3.44) is that we use the notation $\subseteq$ instead of the one $=$, because the right hand side of (3.44) may contain extra diagrams which do not belong to the left hand side. In our example, when we analyze the effective vertex $V_{2}$, it will contain

$$
\left(1-\mathcal{P}_{-, 3}^{+, 2}\right) \operatorname{PT}[(1,2),+,-,(3),(4,5,6,7),(8)] P T[(1,2),+,-,(8),(4,6,5,7),(3)] .
$$

This term contains a 10-point tree diagram as shown in graph B of figure 8, which contributes to $\widehat{m}_{\mathcal{S O}}^{1-\text { loop }}\left[V_{1}\right]$ since it is obtained by cutting an edge of one loop diagram (graph A of figure 8). Furthermore, there are some terms appearing in both (3.43) and (3.44). For example, (3.45) will appear in both $\widehat{m}_{\mathcal{S O}}^{1-\text { loop }}\left[V_{1}\right]$ and $\widehat{m}_{\mathcal{R O}}^{1-\text { loop }}\left[V_{2}\right]$. When we sum contributions from all effective vertexes, these repeated terms should be included only once.

Finally, we move to the problem of how to dislodge massless bubble part. The tree diagrams after cutting a massless bubble are shown in figure 4, from which we see that all

\footnotetext{
${ }^{12} A_{i}^{\prime}$ can not be an arbitrary permutation of $A_{i}$, since they must be connected by some flips at the cubic vertexes.
} 


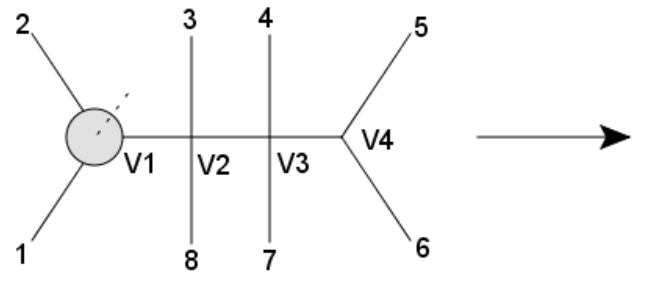

A

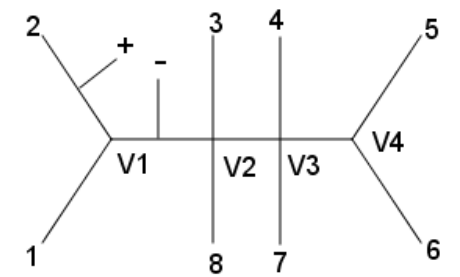

B

Figure 8. The redundancy of two neighbouring effective vertex.

$(n+2)$-point tree diagrams obtained by cutting massless bubbles are included in following type of terms:

$$
P T(\cdots, i,+,-, \cdots) P T(\cdots, i,+,-, \cdots) \text { or } \quad P T(\cdots,+,-, i, \cdots) P T(\cdots,+,-, i, \cdots)
$$

Now we can apply the method of picking out pole to eliminate them. There are two cases. In the first case, all Feynman diagrams produced by a CHY-integrand will contain the $s_{i+-}$ or $s_{+-i}$ poles. For these CHY-integrands, we just remove them from the sum. In the second case, $s_{i+-}$ pole will appear only on some Feynman diagrams (but not all). Thus we need to multiply two cross ratio factor $\mathcal{P} \mathcal{P}$ to pick up these terms as we did in (3.8).

Now we need to understand when all Feynman diagrams produced by a CHY-integrand will contain the $s_{i+-}$ pole. It is easy to see their effective Feynman diagrams will be these in (3.28) and (3.29), thus if using the method given in [3], will be following diagrams
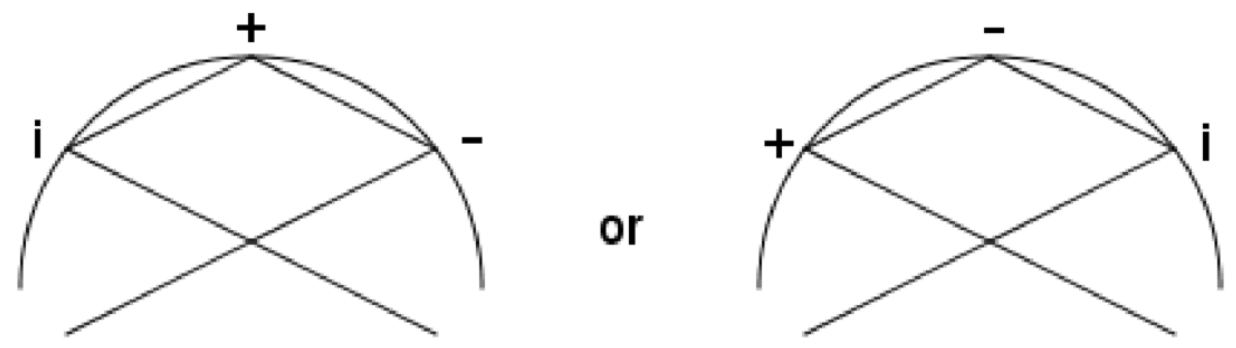

This structure of the left hand graph in (3.47) means that in one PT-factor, the ordering is $(\ldots, a, i,+,-, b, \ldots)$, while in another PT-factor, the ordering is $(\ldots, b, \ldots, i,+,-, \ldots, a, \ldots)$. Similar conclusion holds for the right hand graph. In our 
example with $\pi=(3,4,5,6,7,8,1,2), \rho=(3,1,2,8,4,6,5,7)$. following terms

$$
\begin{array}{r}
P T(1,2, \underline{+,-, 3}, 4,5,6,7,8,9) P T(1,2,8,4,6,5,7, \underline{+,-, 3}) \\
\text { and } P T(1,2, \underline{3,+,-}, 4,5,6,7,8,9) P T(1,2,8,4,6,5,7, \underline{3,+,-}) .
\end{array}
$$

should be removed by our criterion (3.47) for the pole $s_{3+-}$.

From the criterion (3.47), we see directly that all external legs attached to $\mathcal{R O}$-type effective vertexes should be removed while all those attached to $\mathcal{S O}$-type vertex should be kept. In addition, if we attribute those diagrams, where the loop is inserted on an effective propagator, only to the $\mathcal{S} \mathcal{O}$-type vertex attached to the propagator, we can rewrite (3.44) as:

$$
\widehat{m}_{\mathcal{R O}}^{1-\text { loop }}[V]=\sum_{i=1}^{m}\left\{\left(1-\mathcal{P}_{-, \cdot}^{+, \cdot}\right) \sum_{j \neq i, i \pm 1} P T\left(\cdots\left(A_{i}\right),+,-,\left(A_{i+1}\right), \cdots\right) P T\left(\cdots\left(A_{j+1}^{\prime}\right),+,-,\left(A_{j}^{\prime}\right) \cdots\right)\right\} .
$$

Now we can use the notation $=$ since in (3.49) all mentioned problems have been fixed. Moreover, there are no repeated terms appearing in both (3.43) and (3.49). Therefore, (3.39) can be rewrote as

$$
\widehat{m}_{n}^{1-\text { loop }}[\pi \mid \rho]=\sum_{V_{i}} \widehat{m}_{\mathcal{S O} / \mathcal{R O O}}^{1-\text { loop }}\left[V_{i}\right]
$$

Up to now, we have discussed all technical points of our construction. Now we summarize our algorithm for general $m_{n}^{1-\operatorname{loop}}[\pi \mid \rho]$ :

- (1) At the first step, we draw the $n$-point tree-level effective Feynman diagram of $m_{n}^{\text {tree }}[\pi \mid \rho]$.

- (2) For each effective vertex, we determine its relative ordering, i.e., either $\mathcal{S O}$-type or $\mathcal{R O}$-type.

- (3) For each effective vertex, we apply formula (3.43) or (3.49) to get CHY-integrands.

- (4) Now we add those terms coming from different effective vertexes together as given in $(3.50)$.

- (5) For terms from previous step having the pattern in (3.46), we need to remove the massless bubble contributions using the cross ratio factor as did (3.8).

- (6) After finishing above steps, we finally get the CHY-integrand we are looking for.

\subsection{Example}

In this subsection, we present two examples to demonstrate our algorithm. 


\subsubsection{The first example}

The first example is $m_{8}^{1-\text { loop }}[12345678 \mid 12846573]$ with its effective tree Feynman diagram shown in (2.13) where $V_{1}, V_{3}$ are $\mathcal{S O}$-type and $V_{2}, V_{4}, \mathcal{R O}$-type. For $V_{1}$, we have

$$
\begin{aligned}
& \left(1-\mathcal{P}_{-, 2}^{+, 1}\right) P T[(1)+-(2)(345678)] P T[(1)+-(2)(846573)] \\
& \left(1-\mathcal{P}_{-, 3}^{+, 2}\right) P T[(1)(2)+-(345678)] P T[(1)(2)+-(846573)] \\
& \left(1-\mathcal{P}_{-, 1}^{+, 8}\right) P T[(1)(2)(345678)+-] P T[(1)(2)(846573)+-] .
\end{aligned}
$$

For $V_{2}$, we have:

$$
\begin{aligned}
& \left(1-\mathcal{P}_{-, 3}^{+, 2}\right) P T[(12)+-(3)(4567)(8)] P T[(12)(8)+-(4657)(3)] \\
& \left(1-\mathcal{P}_{-, 4}^{+, 3}\right) P T[(12)(3)+-(4567)(8)] P T[(12)+-(8)(4657)(3)] \\
& \left(1-\mathcal{P}_{-, 8}^{+, 7}\right) P T[(12)(3)(4567)+-(8)] P T[(12)(8)(4657)(3)+-] \\
& \left(1-\mathcal{P}_{-, 1}^{+, 8}\right) P T[(12)(3)(4567)(8)+-] P T[(12)(8)(4657)+-(3)]
\end{aligned}
$$

For $V_{3}$, we have:

$$
\begin{aligned}
& \left(1-\mathcal{P}_{-, 4}^{+, 3}\right) \operatorname{PT}[(8123)+-(4)(56)(7)] P T[(3128)+-(4)(65)(7)] \\
& \left(1-\mathcal{P}_{-, 5}^{+, 4}\right) \operatorname{PT}[(8123)(4)+-(56)(7)] P T[(3128)(4)+-(65)(7)] \\
& \left(1-\mathcal{P}_{-, 7}^{+, 6}\right) \operatorname{PT}[(8123)(4)(56)+-(7)] P T[(3128)(4)(65)+-(7)] \\
& \left(1-\mathcal{P}_{-, 8}^{+, 7}\right) \operatorname{PT}[(8123)(4)(56)(7)+-] P T[(3128)(4)(65)(7)+-]
\end{aligned}
$$

For $V_{4}$, we have no contribution. Assembling above, we have 11 terms in total. By applying picking operator $\mathcal{P}$ to terms containing massless bubbles, we obtain:

$$
\begin{aligned}
& m_{8}^{1-\text { loop }}[12345678 \mid 12846573]=\int \frac{d^{D} l}{(2 \pi)^{D}} \frac{1}{l^{2}} \lim _{\text {forward }} \int d \Omega \\
& \times\left\{\left[1-\frac{z_{+1} z_{-2}}{z_{-1} z_{+2}}-\frac{z_{18} z_{+-}}{z_{1-} z_{+8}} \times \frac{z_{18} z_{-2}}{z_{12} z_{-8}}-\frac{z_{-+} z_{23}}{z_{-3} z_{2+}} \times \frac{z_{+1} z_{23}}{z_{+3} z_{21}}\right] P T(1+-2345678) P T(1+-2846573)\right. \\
& \quad+\left[1-\frac{z_{+2} z_{-3}}{z_{+3} z_{-2}}-\frac{z_{21} z_{+-}}{z_{2-} z_{+1}} \times \frac{z_{21} z_{-3}}{z_{23} z_{-1}}\right] P T(12+-345678) P T(12+-846573) \\
& \quad+\left[1-\frac{z_{+8} z_{-1}}{z_{+1} z_{-8}}-\frac{z_{-+} z_{12}}{z_{-2} z_{1+}} \times \frac{z_{+8} z_{12}}{z_{+2} z_{18}}\right] P T(12345678+-) P T(12846573+-) \\
& \quad+\left[1-\frac{z_{+2} z_{-3}}{z_{+3} z_{-2}}\right] P T(12+-345678) P T(128+-46573) \\
& \quad+\left[1-\frac{z_{+3} z_{-4}}{z_{+4} z_{-3}}\right] P T(123+-45678) P T(12+-846573) \\
& \quad+\left[1-\frac{z_{+3} z_{-4}}{z_{+4} z_{-3}}-\frac{z_{-+} z_{45}}{z_{-5} z_{4+}} \times \frac{z_{+3} z_{45}}{z_{+5} z_{43}}\right] P T(123+-45678) P T(128+-46573) \\
& \quad+\left[1-\frac{z_{+7} z_{-8}}{z_{+8} z_{-7}}-\frac{z_{76} z_{+-}}{z_{7-} z_{+6}} \times \frac{z_{76} z_{-8}}{z_{78} z_{-6}}\right] P T(1234567+-8) P T(1284657+-3) \\
& \quad+\left[1-\frac{z_{+7} z_{-8}}{z_{+8} z_{-7}}\right] P T(1234567+-8) P T(12846573+-) \\
& \quad+\left[1-\frac{z_{+8} z_{-1}}{z_{+1} z_{-8}}\right] P T(12345678+-) P T(1284657+-3)
\end{aligned}
$$




$$
\begin{aligned}
& +\left[1-\frac{z_{+4} z_{-5}}{z_{+5} z_{-4}}-\frac{z_{43} z_{+-}}{z_{4-} z_{+3}} \times \frac{z_{43} z_{-5}}{z_{45} z_{-3}}\right] P T(1234+-5678) P T(1284+-6573) \\
& \left.+\left[1-\frac{z_{+6} z_{-7}}{z_{+7} z_{-6}}-\frac{z_{-+} z_{78}}{z_{-8} z_{7+}} \times \frac{z_{+6} z_{78}}{z_{+8} z_{76}}\right] P T(123456+-78) P T(128465+-73)\right\} .
\end{aligned}
$$

Compared it with construction (3.5) with 64 terms, the difference it exactly those massless bubbles.

\subsubsection{The second example}

The second example is $m_{9}^{1-\text { loop }}[123456789 \mid 127893654]$ with its effective tree Feynman diagram given by

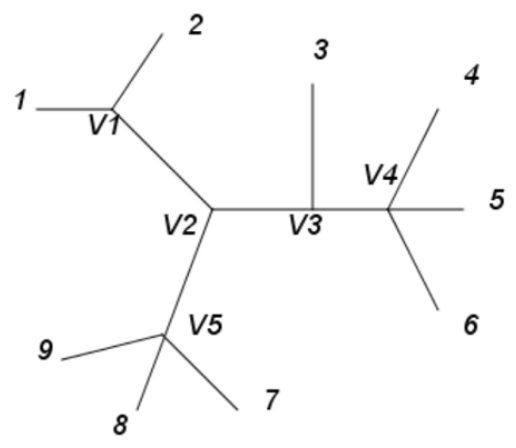

where $V_{1}, V_{3}, V_{5}$ are $\mathcal{S O}$-type and $V_{2}, V_{4}, \mathcal{R O}$-type. For $V_{1}$, we have

$$
\begin{aligned}
& \left(1-\mathcal{P}_{-, 2}^{+, 1}\right) P T[(1)+-(2)(3456789)] P T[(1)+-(2)(7893654)] \\
& \left(1-\mathcal{P}_{-, 3}^{+, 2}\right) P T[(1)(2)+-(3456789)] P T[(1)(2)+-(7893654)] \\
& \left(1-\mathcal{P}_{-, 1}^{+, 9}\right) P T[(1)(2)(3456789)+-] P T[(1)(2)(7893654)+-] .
\end{aligned}
$$

For $V_{2}$, we have no contribution. For $V_{3}$ we have:

$$
\begin{aligned}
& \left(1-\mathcal{P}_{-, 3}^{+, 2}\right) P T[(78912)+-(3)(456)] P T[(12789)+-(3)(654)] \\
& \left(1-\mathcal{P}_{-, 4}^{+, 3}\right) P T[(78912)(3)+-(456)] P T[(12789)(3)+-(654)] \\
& \left(1-\mathcal{P}_{-, 7}^{+, 6}\right) P T[(78912)(3)(456)+-] P T[(12789)(3)(654)+-] .
\end{aligned}
$$

For $V_{4}$, we have:

$$
\begin{aligned}
& \left(1-\mathcal{P}_{-, 4}^{+, 3}\right) P T[(789123)+-(4)(5)(6)] P T[(127893)(6)+-(5)(4)] \\
& \left(1-\mathcal{P}_{-, 5}^{+, 4}\right) P T[(789123)(4)+-(5)(6)] P T[(127893)+-(6)(5)(4)] \\
& \left(1-\mathcal{P}_{-, 6}^{+, 5}\right) P T[(789123)(4)(5)+-(6)] P T[(127893)(6)(5)(4)+-] \\
& \left(1-\mathcal{P}_{-, 7}^{+, 6}\right) P T[(789123)(4)(5)(6)+-] P T[(127893)(6)(5)+-(4)] .
\end{aligned}
$$


For $V_{5}$, we have:

$$
\begin{aligned}
& \left(1-\mathcal{P}_{-, 7}^{+, 6}\right) P T[(123456)+-(7)(8)(9)] P T[(365412)+-(7)(8)(9)] \\
& \left(1-\mathcal{P}_{-, 8}^{+, 7}\right) P T[(123456)(7)+-(8)(9)] P T[(365412)(7)+-(8)(9)] \\
& \left(1-\mathcal{P}_{-, 9}^{+, 8}\right) P T[(123456)(7)(8)+-(9)] P T[(365412)(7)(8)+-(9)] \\
& \left(1-\mathcal{P}_{-, 1}^{+, 9}\right) P T[(123456)(7)(8)(9)+-] P T[(365412)(7)(8)(9)+-] .
\end{aligned}
$$

Assembling above, we have 14 terms in total. By applying picking operator $\mathcal{P}$ to massless bubbles, we obtain:

$$
\begin{aligned}
& m_{9}^{1-\text { loop }}[123456789 \mid 127893654]=\int \frac{d^{D} l}{(2 \pi)^{D}} \frac{1}{l^{2}} \lim _{\text {forward }} \int d \Omega \\
& \times\left\{\left[1-\frac{z_{+1} z_{-2}}{z_{-1} z_{+2}}-\frac{z_{19} z_{+-}}{z_{1-} z_{+9}} \times \frac{z_{19} z_{-2}}{z_{12} z_{-9}}-\frac{z_{-+} z_{23}}{z_{-3} z_{2+}} \times \frac{z_{+1} z_{23}}{z_{+3} z_{21}}\right] P T(1+-23456789) P T(1+-27893654)\right. \\
& +\left[1-\frac{z_{+2} z_{-3}}{z_{+3} z_{-2}}-\frac{z_{21} z_{+-}}{z_{2-} z_{+1}} \times \frac{z_{21} z_{-3}}{z_{23} z_{-1}}\right] P T(12+-3456789) P T(12+-7893654) \\
& +\left[1-\frac{z_{+9} z_{-1}}{z_{+1} z_{-9}}-\frac{z_{-+} z_{12}}{z_{-2} z_{1+}} \times \frac{z_{+9} z_{12}}{z_{+2} z_{19}}\right] P T(123456789+-) P T(127893654+-) \\
& +\left[1-\frac{z_{+2} z_{-3}}{z_{+3} z_{-2}}-\frac{z_{-+} z_{34}}{z_{-4} z_{3+}} \times \frac{z_{+9} z_{34}}{z_{+4} z_{39}}\right] P T(12+-3456789) P T(12789+-3654) \\
& +\left[1-\frac{z_{+3} z_{-4}}{z_{+4} z_{-3}}-\frac{z_{32} z_{+-}}{z_{3-} z_{+2}} \times \frac{z_{32} z_{-4}}{z_{34} z_{-2}}\right] P T(123+-456789) P T(127893+-654) \\
& +\left[1-\frac{z_{+6} z_{-7}}{z_{+7} z_{-6}}\right] P T(123456+-789) P T(127893654+-) \\
& +\left[1-\frac{z_{+3} z_{-4}}{z_{+4} z_{-3}}\right] P T(123+-456789) P T(1278936+-54) \\
& +\left[1-\frac{z_{+4} z_{-5}}{z_{+5} z_{-4}}\right] P T(1234+-56789) P T(127893+-654) \\
& +\left[1-\frac{z_{+5} z_{-6}}{z_{+6} z_{-5}}\right] P T(12345+-6789) P T(127893654+-) \\
& +\left[1-\frac{z_{+6} z_{-7}}{z_{+7} z_{-6}}\right] P T(123456+-789) P T(12789365+-4) \\
& +\left[1-\frac{z_{+6} z_{-7}}{z_{+7} z_{-6}}-\frac{z_{-+} z_{78}}{z_{-8} z_{7+}} \times \frac{z_{+6} z_{78}}{z_{+8} z_{76}}\right] P T(123456+-789) P T(12+-7893654) \\
& +\left[1-\frac{z_{+7} z_{-8}}{z_{-8} z_{+7}}-\frac{z_{76} z_{+-}}{z_{7-} z_{+6}} \times \frac{z_{76} z_{-8}}{z_{78} z_{-6}}-\frac{z_{-+} z_{89}}{z_{-9} z_{8+}} \times \frac{z_{+7} z_{89}}{z_{+9} z_{87}}\right] P T(1234567+-89) P T(127+-893654) \\
& +\left[1-\frac{z_{+8} z_{-9}}{z_{-9} z_{+8}}-\frac{z_{87} z_{+-}}{z_{8-} z_{+7}} \times \frac{z_{87} z_{-9}}{z_{89} z_{-7}}-\frac{z_{-+} z_{91}}{z_{-1} z_{9+}} \times \frac{z_{+8} z_{91}}{z_{+1} z_{98}}\right] P T(12345678+-9) P T(1278+-93654) \\
& \left.+\left[1-\frac{z_{+9} z_{-1}}{z_{-9} z_{+1}}-\frac{z_{98} z_{+-}}{z_{9-} z_{+8}} \times \frac{z_{98} z_{-1}}{z_{91} z_{-8}}-\frac{z_{-+} z_{12}}{z_{-2} z_{1+}} \times \frac{z_{+9} z_{12}}{z_{+2} z_{19}}\right] P T(123456789+-) P T(12789+-3654)\right\} .
\end{aligned}
$$

Compared it with construction (3.5) with 81 terms, the difference is exactly those external bubbles. 


\section{Conclusion}

In this paper, we have focused on the construction of one-loop CHY-integrands for biadjoint scalar theory. Our construction is different from the one given in $[18,26]$ by the treatment of singularities related to the forward limit. Now it is well known that one can construct one-loop amplitudes from tree's by taking the forward limits. However, in the forward limit, some singularities as well as extra terms will appear, such as the tadpoles and massless bubbles, which are not considered for the standard amputated oneloop Feynman diagrams. Different treatments of singularities and extra terms as well as the singular solutions from scattering equations will give different construction of one-loop CHY-integrands, although all of them are in the same equivalent class. Our new strategy in this paper is to use the picking pole operators to remove the singularities and extra terms explicitly. More explicitly, by exploiting the concept of effective Feynman diagrams, we show how to find corresponding tree-level diagrams and how to remove singular tadpoles and massless bubbles by using the cross ratio factor defined in [37].

The reason we investigate the new strategy is to hope that such a method can be generalized to more general theories appearing in the CHY formalism, such as Yang-Mills theories, NLSM etc. It has been established that all theories can be expanded to bi-adjoint scaler theory [46]. For example, in [48] the expansion of Pfaffian to the combination of PT factors has been presented. Thus Yang-Mills CHY-integrand can be written as the sum of bi-adjoint scalar theories. Using results in this paper, we remove all singularities and extra terms for each bi-adjoint CHY-integrand in the expansion. Now we end up a sum without any singularities by the brute force way, but is it the right result? Checking this idea is the future project we are working on. Furthermore, even above thought works, expansion to PT factors is very complicated, thus looking for a better way to remove singularities and extra terms along the line of this paper is also intriguing questions to ask.

\section{Acknowledgments}

We would like to thank E. Y. Yuan for discussion and reading of draft. This work is supported by Qiu-Shi Funding and Chinese NSF funding under Grant No.11575156, No.11935013.

Open Access. This article is distributed under the terms of the Creative Commons Attribution License (CC-BY 4.0), which permits any use, distribution and reproduction in any medium, provided the original author(s) and source are credited.

\section{References}

[1] F. Cachazo, S. He and E.Y. Yuan, Scattering equations and Kawai-Lewellen-Tye orthogonality, Phys. Rev. D 90 (2014) 065001 [arXiv:1306.6575] [INSPIRE].

[2] F. Cachazo, S. He and E.Y. Yuan, Scattering of massless particles in arbitrary dimensions, Phys. Rev. Lett. 113 (2014) 171601 [arXiv:1307.2199] [InSPIRE]. 
[3] F. Cachazo, S. He and E.Y. Yuan, Scattering of massless particles: scalars, gluons and gravitons, JHEP 07 (2014) 033 [arXiv:1309.0885] [InSPIRE].

[4] F. Cachazo, S. He and E.Y. Yuan, Einstein-Yang-Mills scattering amplitudes from scattering equations, JHEP 01 (2015) 121 [arXiv:1409.8256] [INSPIRE].

[5] F. Cachazo, S. He and E.Y. Yuan, Scattering equations and matrices: from Einstein to Yang-Mills, DBI and NLSM, JHEP 07 (2015) 149 [arXiv:1412.3479] [INSPIRE].

[6] L. Dolan and P. Goddard, Proof of the formula of Cachazo, He and Yuan for Yang-Mills tree amplitudes in arbitrary dimension, JHEP 05 (2014) 010 [arXiv:1311.5200] [INSPIRE].

[7] L. Dolan and P. Goddard, The polynomial form of the scattering equations, JHEP 07 (2014) 029 [arXiv: 1402.7374] [INSPIRE].

[8] C. Kalousios, Scattering equations, generating functions and all massless five point tree amplitudes, JHEP 05 (2015) 054 [arXiv: 1502.07711] [INSPIRE].

[9] R. Huang, J. Rao, B. Feng and Y.-H. He, An algebraic approach to the scattering equations, JHEP 12 (2015) 056 [arXiv: 1509. 04483] [INSPIRE].

[10] M. Søgaard and Y. Zhang, Scattering equations and global duality of residues, Phys. Rev. D 93 (2016) 105009 [arXiv: 1509.08897] [INSPIRE].

[11] L. Dolan and P. Goddard, General solution of the scattering equations, JHEP 10 (2016) 149 [arXiv: 1511.09441] [INSPIRE].

[12] C. Cardona and C. Kalousios, Comments on the evaluation of massless scattering, JHEP 01 (2016) 178 [arXiv: 1509.08908] [inSPIRE].

[13] C. Cardona and C. Kalousios, Elimination and recursions in the scattering equations, Phys. Lett. B 756 (2016) 180 [arXiv:1511.05915] [INSPIRE].

[14] F. Cachazo and H. Gomez, Computation of contour integrals on $\mathcal{M}_{0, n}$, JHEP 04 (2016) 108 [arXiv: 1505.03571] [INSPIRE].

[15] F. Cachazo, K. Yeats and S. Yusim, Compatible cycles and CHY integrals, JHEP 12 (2019) 105 [arXiv: 1907.12661] [INSPIRE].

[16] C. Baadsgaard, N.E.J. Bjerrum-Bohr, J.L. Bourjaily and P.H. Damgaard, Integration rules for scattering equations, JHEP 09 (2015) 129 [arXiv: 1506. 06137] [INSPIRE].

[17] C. Baadsgaard, N.E.J. Bjerrum-Bohr, J.L. Bourjaily and P.H. Damgaard, Scattering equations and Feynman diagrams, JHEP 09 (2015) 136 [arXiv:1507.00997] [INSPIRE].

[18] C. Baadsgaard, N.E.J. Bjerrum-Bohr, J.L. Bourjaily, P.H. Damgaard and B. Feng, Integration rules for loop scattering equations, JHEP 11 (2015) 080 [arXiv:1508.03627] [INSPIRE].

[19] C. Cardona, B. Feng, H. Gomez and R. Huang, Cross-ratio identities and higher-order poles of CHY-integrand, JHEP 09 (2016) 133 [arXiv:1606.00670] [INSPIRE].

[20] C.S. Lam and Y.-P. Yao, Evaluation of the Cachazo-He-Yuan gauge amplitude, Phys. Rev. D 93 (2016) 105008 [arXiv: 1602.06419] [INSPIRE].

[21] T. Adamo, E. Casali and D. Skinner, Ambitwistor strings and the scattering equations at one loop, JHEP 04 (2014) 104 [arXiv: 1312.3828] [INSPIRE].

[22] E. Casali and P. Tourkine, Infrared behaviour of the one-loop scattering equations and supergravity integrands, JHEP 04 (2015) 013 [arXiv: 1412.3787] [INSPIRE]. 
[23] T. Adamo and E. Casali, Scattering equations, supergravity integrands and pure spinors, JHEP 05 (2015) 120 [arXiv: 1502.06826] [INSPIRE].

[24] Y. Geyer, L. Mason, R. Monteiro and P. Tourkine, Loop integrands for scattering amplitudes from the Riemann sphere, Phys. Rev. Lett. 115 (2015) 121603 [arXiv:1507.00321] [INSPIRE].

[25] Y. Geyer, L. Mason, R. Monteiro and P. Tourkine, One-loop amplitudes on the Riemann sphere, JHEP 03 (2016) 114 [arXiv:1511.06315] [INSPIRE].

[26] S. He and E.Y. Yuan, One-loop scattering equations and amplitudes from forward limit, Phys. Rev. D 92 (2015) 105004 [arXiv: 1508.06027] [INSPIRE].

[27] F. Cachazo, S. He and E.Y. Yuan, One-loop corrections from higher dimensional tree amplitudes, JHEP 08 (2016) 008 [arXiv:1512.05001] [INSPIRE].

[28] M. Zlotnikov, Polynomial reduction and evaluation of tree- and loop-level CHY amplitudes, JHEP 08 (2016) 143 [arXiv: 1605.08758] [INSPIRE].

[29] C. Cardona and H. Gomez, CHY-graphs on a torus, JHEP 10 (2016) 116 [arXiv: 1607.01871] [INSPIRE].

[30] S. He and O. Schlotterer, New relations for gauge-theory and gravity amplitudes at loop level, Phys. Rev. Lett. 118 (2017) 161601 [arXiv:1612.00417] [INSPIRE].

[31] H. Gomez, S. Mizera and G. Zhang, CHY loop integrands from holomorphic forms, JHEP 03 (2017) 092 [arXiv: 1612.06854] [INSPIRE].

[32] H. Gomez, Quadratic Feynman loop integrands from massless scattering equations, Phys. Rev. D 95 (2017) 106006 [arXiv: 1703.04714] [INSPIRE].

[33] H. Gomez, C. Lopez-Arcos and P. Talavera, One-loop Parke-Taylor factors for quadratic propagators from massless scattering equations, JHEP 10 (2017) 175 [arXiv:1707.08584] [INSPIRE].

[34] Y. Geyer and R. Monteiro, Gluons and gravitons at one loop from ambitwistor strings, JHEP 03 (2018) 068 [arXiv:1711.09923] [INSPIRE].

[35] N. Ahmadiniaz, H. Gomez and C. Lopez-Arcos, Non-planar one-loop Parke-Taylor factors in the CHY approach for quadratic propagators, JHEP 05 (2018) 055 [arXiv:1802.00015] [INSPIRE].

[36] J. Agerskov, N.E.J. Bjerrum-Bohr, H. Gomez and C. Lopez-Arcos, Yang-Mills loop amplitudes from scattering equations, arXiv:1910.03602 [INSPIRE].

[37] B. Feng, CHY-construction of planar loop integrands of cubic scalar theory, JHEP 05 (2016) 061 [arXiv: 1601.05864 ] [INSPIRE].

[38] Y. Geyer, L. Mason, R. Monteiro and P. Tourkine, Two-loop scattering amplitudes from the Riemann sphere, Phys. Rev. D 94 (2016) 125029 [arXiv:1607.08887] [InSPIRE].

[39] Y. Geyer and R. Monteiro, Two-loop scattering amplitudes from ambitwistor strings: from genus two to the nodal Riemann sphere, JHEP 11 (2018) 008 [arXiv:1805.05344] [INSPIRE].

[40] Y. Geyer, R. Monteiro and R. Stark-Muchão, Two-loop scattering amplitudes: double-forward limit and colour-kinematics duality, JHEP 12 (2019) 049 [arXiv: 1908.05221] [INSPIRE].

[41] R. Huang, B. Feng, M.-X. Luo and C.-J. Zhu, Feynman rules of higher-order poles in CHY construction, JHEP 06 (2016) 013 [arXiv: 1604.07314] [INSPIRE]. 
[42] R. Huang, Y.-J. Du and B. Feng, Understanding the cancelation of double poles in the Pfaffian of CHY-formulism, JHEP 06 (2017) 133 [arXiv:1702.05840] [INSPIRE].

[43] R. Huang, F. Teng and B. Feng, Permutation in the CHY-formulation, Nucl. Phys. B 932 (2018) 323 [arXiv : 1801.08965] [InSPIRE].

[44] C. Baadsgaard, Amplitudes from sting theory and CHY formalism, master's thesis, Copenhagen University, Copenhagen, Denmark (2015).

[45] N.E.J. Bjerrum-Bohr, J.L. Bourjaily, P.H. Damgaard and B. Feng, Analytic representations of Yang-Mills amplitudes, Nucl. Phys. B 913 (2016) 964 [arXiv:1605.06501] [inSPIRE].

[46] N.E.J. Bjerrum-Bohr, J.L. Bourjaily, P.H. Damgaard and B. Feng, Manifesting color-kinematics duality in the scattering equation formalism, JHEP 09 (2016) 094 [arXiv: 1608.00006] [INSPIRE].

[47] K. Zhou, J. Rao and B. Feng, Derivation of Feynman rules for higher order poles using cross-ratio identities in CHY construction, JHEP 06 (2017) 091 [arXiv:1705.04783] [INSPIRE].

[48] C.-H. Fu, Y.-J. Du, R. Huang and B. Feng, Expansion of Einstein-Yang-Mills amplitude, JHEP 09 (2017) 021 [arXiv:1702.08158] [INSPIRE]. 